\title{
Rapid contraction of giant planets orbiting the 20-million-year-old star V1298 Tau
}

\author{
A. Suárez Mascareño ${ }^{1,2} \llbracket$, M. Damasso3 ${ }^{3}$ N. Lodieu ${ }^{1,2}$, A. Sozzetti ${ }^{3}$, V. J. S. Béjar ${ }^{1,2}$, S. Benatti ${ }^{4}$, \\ M. R. Zapatero Osorio ${ }^{5}{ }^{5}$, G. Micela ${ }^{4}$, R. Rebolo ${ }^{1,2,6}$, S. Desidera7, F. Murgas ${ }^{1,2}$, R. Claudi ${ }^{7}$, \\ J. I. González Hernández ${ }^{1,2}$, L. Malavolta ${ }^{7,8}$, C. del Burgo9 , V. D'Orazi ${ }^{7,10}$, P. J. Amado ${ }^{11}$, \\ D. Locci ${ }^{4}$, H. M. Tabernero ${ }^{5,12}$, F. Marzari ${ }^{8}$, D. S. Aguado ${ }^{13,14}$, D. Turrini ${ }^{3}{ }^{3}$, C. Cardona Guillén ${ }^{1,2}$, \\ B. Toledo-Padrón'1,2, A. Maggio ${ }^{4}{ }^{4}$, J. Aceituno ${ }^{11,15}$, F. F. Bauer11, J. A. Caballero ${ }^{16}{ }^{16}$, P. Chinchilla ${ }^{1,17}$, \\ E. Esparza-Borges $\mathbb{1}^{1,2}$, E. González-Álvarez ${ }^{5}$, T. Granzer ${ }^{18}$, R. Luque ${ }^{11}{ }^{11}$, E. L. Martín ${ }^{1,2,6}$, \\ G. Nowak $\mathbb{1}^{1,2}$, M. Oshagh $\mathbb{1}^{1,2}$, E. Pallé1,2, H. Parviainen ${ }^{1,2}$, A. Quirrenbach ${ }^{19}$, A. Reiners $\mathbb{1}^{20}$, \\ I. Ribas ${ }^{21,22}$, K. G. Strassmeier ${ }^{18}$, M. Weber ${ }^{18}$ and M. Mallonn ${ }^{18}$
}

\begin{abstract}
Current theories of planetary evolution predict that infant giant planets have large radii and very low densities before they slowly contract to reach their final size after about several hundred million years ${ }^{1,2}$. These theoretical expectations remain untested so far as the detection and characterization of very young planets is extremely challenging due to the intense stellar activity of their host stars ${ }^{3,4}$. Only the recent discoveries of young planetary transiting systems allow initial constraints to be placed on evolutionary models ${ }^{5-7}$. With an estimated age of $\mathbf{2 0}$ million years, V1298 Tau is one of the youngest solar-type stars known to host transiting planets; it harbours a system composed of four planets, two Neptune-sized, one Saturn-sized and one Jupiter-sized ${ }^{8,9}$. Here we report a multi-instrument radial velocity campaign of V1298 Tau, which allowed us to determine the masses of two of the planets in the system. We find that the two outermost giant planets, V1298 Tau $b$ and e $(0.64 \pm 0.19$ and $1.16 \pm 0.30$ Jupiter masses, respectively), seem to contradict our knowledge of early-stages planetary evolution. According to models, they should reach their mass-radius combination only hundreds of millions of years after formation. This result suggests that giant planets can contract much more quickly than usually assumed.
\end{abstract}

V1298 Tau is a relatively bright $(V=10.1)$, very young $\mathrm{K} 1$ star with a mass of $1.170 \pm 0.060 M_{\odot}$ (where $M_{\odot}$ is the solar mass), a radius of $1.278 \pm 0.070 R_{\odot}$ (where $R_{\odot}$ is the solar radius), an effective temperature of $5,050 \pm 100 \mathrm{~K}$ and solar metallicity (Table 1 and Extended Data Fig. 1). It is the physical companion of the G2 star HD 284154. The pair belongs to the Group 29 stellar association ${ }^{10}$ and has an age of $20 \pm 10$ Myr (Extended Data Figs. 1 and 2). V1298 Tau was observed by Kepler's 'Second Light' K2 mission ${ }^{11}$. The analysis of the K2 data revealed the presence of four transiting planets in the system ${ }^{9}$. The three inner planets (b, c and d) were determined to have orbital periods of $24.1396 \pm 0.0018,8.24958 \pm 0.00072$ and $12.4032 \pm 0.0015$ days, and radii of $0.916_{-0.047}^{+0.052}, 0.499_{-0.029}^{+0.032}$ and $0.572_{-0.035}^{+0.040} R_{\text {Jup }}$ (where $R_{\text {Jup }}$ is the Jupiter radius). The fourth planet, $\mathrm{e}$, was identified with only a single transit event, with a radius of $0.780_{-0.064}^{+0.075} R_{\text {Jup }}$ and orbital period estimated to be between 40 and 120 days. A previous study constrained the mass of V1298 Tau b to be less than $2.2 M_{\text {Jup }}$ (ref. ${ }^{12}$ ) (where $M_{\text {Jup }}$ is the Jupiter mass).

To measure the planetary masses, we performed an intensive spectroscopic campaign, collecting more than 260 radial velocity (RV) measurements of V1298 Tau using the high-resolution spectrographs HARPS-N, CARMENES, SES and HERMES between April 2019 and April 2020. To monitor its stellar activity variations, we performed contemporaneous $V$-band photometry using the Las Cumbres Observatory Global Telescope (LCOGT) network ${ }^{13}$.

V1298 Tau is a very active star that induces large RV activity variations. To extract the planetary signals in the RV we opted for a global model combining the K2 photometry, the RV and the LCOGT photometry. We relied on a Gaussian processes regression ${ }^{14}$ to account for the stellar activity contribution. The model uses,

\footnotetext{
'Instituto de Astrofísica de Canarias, La Laguna, Spain. ${ }^{2}$ Departamento de Astrofisica, Universidad de La Laguna, La Laguna, Spain. ${ }^{3}$ sstituto Nazionale di Astrofisica - Osservatorio Astrofisico di Torino, Pino Torinese, Italy. ${ }^{4}$ Istituto Nazionale di Astrofisica - Osservatorio Astronomico di Palermo, Palermo, Italy. ${ }^{5}$ Centro de Astrobiología, Instituto Nacional de Técnica Aeroespacial (Consejo Superior de Investigaciones Científicas), Torrejón de Ardoz, Spain. ${ }^{6}$ Consejo Superior de Investigaciones Científicas, Madrid, Spain. ${ }^{7}$ Istituto Nazionale di Astrofisica - Osservatorio Astronomico di Padova, Padua, Italy. ${ }^{8}$ Dipartimento di Fisica e Astronomia 'Galileo Galilei', Universitá degli Studi di Padova, Padua, Italy. 'Instituto Nacional de Astrofísica, Óptica y Electrónica, Puebla, Mexico. ${ }^{10}$ School of Physics and Astronomy, Monash University, Melbourne, Victoria, Australia. "Instituto de Astrofísica de Andalucía (Consejo Superior de Investigaciones Científicas), Granada, Spain. ${ }^{12}$ Instituto de Astrofísica e Ciências do Espaço, Centro de Astrofísica da Universidade do Porto, Universidade do Porto, Porto, Portugal. ${ }^{13}$ Institute of Astronomy, University of Cambridge, Cambridge, UK. ${ }^{14}$ Dipartimento di Fisica e Astronomia, Universitá degli Studi di Firenze, Sesto Fiorentino, Italy. ${ }^{15}$ Centro Astronómico Hispano-Alemán, Gérgal, Spain. ${ }^{16}$ Centro de Astrobiología, Instituto Nacional de Técnica Aeroespacial (Consejo Superior de Investigaciones Científicas, ESAC), Villanueva de la Cañada, Spain. ${ }^{17}$ Astrobiology Research Unit, Université de Liège, Liège, Belgium. ${ }^{18}$ Leibniz-Institute for Astrophysics Potsdam, Potsdam, Germany. ${ }^{19}$ Landessternwarte, Zentrum für Astronomie der Universität Heidelberg, Heidelberg, Germany. ${ }^{20}$ Institut für Astrophysik, Georg-August-Universität Göttingen, Göttingen, Germany. ${ }^{21}$ Institut de Ciències de I'Espai (Instituto de Ciencias del Espacio) (Consejo Superior de Investigaciones Científicas), Bellaterra, Spain. ${ }^{22}$ Institut d'Estudis Espacials de Catalunya, Barcelona, Spain. 凶e-mail: asm@iac.es
} 


\begin{tabular}{|c|c|c|c|}
\hline Parameter & V1298 Tau & HD 284154 & Reference \\
\hline RA (hms) & 040519.59 & 040514.35 & 85 \\
\hline Dec. $\left({ }^{\prime} \prime^{\prime \prime}\right)$ & +200925.6 & +200821.5 & 85 \\
\hline $\begin{array}{l}\text { Proper motion } \mu_{\alpha} \\
\cos \delta\left(\text { mas } \mathrm{yr}^{-1}\right)\end{array}$ & $5.23 \pm 0.13$ & $5.04 \pm 0.12$ & 85 \\
\hline $\begin{array}{l}\text { Proper motion } \mu_{\delta} \\
\left(\text { mas } \mathrm{yr}^{-1}\right)\end{array}$ & $-16.08 \pm 0.05$ & $-16.32 \pm 0.05$ & 86 \\
\hline Parallax (mas) & $9.258 \pm 0.020$ & $9.290 \pm 0.023$ & 85 \\
\hline Distance $(p c)$ & $108.5 \pm 0.7$ & $108.7 \pm 0.7$ & 85 \\
\hline Spectral type & K1 & $\mathrm{G} 2$ & 87,88 \\
\hline Magnitude V (mag) & $10.12 \pm 0.05$ & $8.51 \pm 0.02$ & 89 \\
\hline Magnitude G (mag) & $10.0702 \pm 0.0007$ & $8.3561 \pm 0.0005$ & 85 \\
\hline Magnitude J (mag) & $8.687 \pm 0.023$ & $7.287 \pm 0.020$ & 90 \\
\hline Magnitude $K_{s}$ (mag) & $8.094 \pm 0.021$ & $6.947 \pm 0.026$ & 90 \\
\hline Velocity $U\left(\mathrm{~km} \mathrm{~s}^{-1}\right)$ & $-12.63 \pm 0.03$ & $-12.90 \pm 0.64$ & This work \\
\hline Velocity $V\left(\mathrm{~km} \mathrm{~s}^{-1}\right)$ & $-6.32 \pm 0.06$ & $-6.32 \pm 0.10$ & This work \\
\hline Velocity W $\left(\mathrm{km} \mathrm{s}^{-1}\right)$ & $-9.19 \pm 0.06$ & $-9.49 \pm 0.28$ & This work \\
\hline Age (Myr) & $20 \pm 10$ & $20 \pm 10$ & This work \\
\hline Luminosity $\left(L_{\odot}\right)$ & $0.954 \pm 0.040$ & $4.138 \pm 0.040^{a}$ & This work \\
\hline $\begin{array}{l}\text { Effective } \\
\text { temperature (K) }\end{array}$ & $5050 \pm 100$ & $5700 \pm 100$ & This work \\
\hline $\operatorname{Mass}\left(M_{\odot}\right)$ & $1.170 \pm 0.060$ & $1.28 \pm 0.06^{b}$ & This work \\
\hline Radius $\left(R_{\odot}\right)$ & $1.278 \pm 0.070$ & $1.477 \pm 0.082^{b}$ & This work \\
\hline Rotation period (d) & $2.91 \pm 0.05$ & $\ldots$ & This work \\
\hline $\begin{array}{l}\text { Rotational velocity } v \\
\sin i\left(\mathrm{~km} \mathrm{~s}^{-1}\right)\end{array}$ & $23.8 \pm 0.5$ & $10 \pm 1$ & This work \\
\hline$[\mathrm{Fe} / \mathrm{H}](\mathrm{dex})$ & $0.10 \pm 0.15$ & $0.05 \pm 0.15$ & This work \\
\hline
\end{tabular}

$i$ corresponds to the inclination angle of the rotation axis with respect to our line of sight. ${ }^{a}$ Corresponds to the binary system. ${ }^{b}$ Measurement for each individual component assuming an equal-mass binary.

simultaneously, the K2 photometry and RVs to constrain the planetary parameters, and the LCOGT photometry and RVs to constrain the timescales and amplitudes of the activity variations during our observing campaign.

We obtained significant measurements of the RV semi-amplitudes induced by planet $b$ of $41 \pm 12 \mathrm{~m} \mathrm{~s}^{-1}$ and by planet e of $62 \pm 16 \mathrm{~m} \mathrm{~s}^{-1}$. For the two innermost planets, $c$ and d, we could only set upper limits of $22 \mathrm{~m} \mathrm{~s}^{-1}$ and $25 \mathrm{~m} \mathrm{~s}^{-1}$ with a $99.7 \%$ confidence, respectively (Extended Data Fig. 4). For planet e we obtained a detection at a period of $40.2 \pm 1.0 \mathrm{~d}$, which is on the short end of the range expected from the transit duration ${ }^{15}$. We measured the orbital eccentricities for planets $b$ and e to be $0.13 \pm 0.07$ and $0.10 \pm 0.09$, respectively. Figure 1 shows the phase-folded curves of the RV signals attributed to planets $b$ and e. The analysis of the RV and photometry during the 2019-2020 campaign yielded a stellar rotation period of $2.9104 \pm 0.0019 \mathrm{~d}$, with a semi-amplitude in RV of about $250 \mathrm{~m} \mathrm{~s}^{-1}$ and of $5 \%$ of the flux in the $V$-band photometry. Our analysis yielded orbital periods, times of transit and relative radii that were consistent with those of the discovery paper. ${ }^{8}$ Supplementary Table 1 and Extended Data Figs. 5-10 show the complete summary of our results. We note that the determination of the period of planet e comes almost exclusively from the spectroscopic analysis, which creates some uncertainty in the origin of the signal. New observations by the Transiting Exoplanet Survey Satellite (TESS) of V1298
Tau (Sectors 43 and 44, September-November 2021) will provide an opportunity to detect a new transit of planet e, further solidifying its orbital period. Considering a typical TESS duty cycle, we estimate a $20 \%$ probability of missing any transit and the same probability of detecting two transits.

We derived the masses of planets $b$ and e to be $0.64 \pm 0.19 M_{\text {Jup }}$ and $1.16 \pm 0.30 M_{\text {Jup }}$, respectively. For the same planets we derived radii of $0.868 \pm 0.056 R_{\text {Jup }}$ and $0.735 \pm 0.072 R_{\text {Jup }}$, respectively, which are compatible with previous measurements ${ }^{8}$. We derived densities of $1.20 \pm 0.45 \mathrm{~g} \mathrm{~cm}^{-3}$ and $3.6 \pm 1.6 \mathrm{~g} \mathrm{~cm}^{-3}$, respectively. V1298 Tau b occupies a position in the mass-radius diagram compatible with the old giant planets of the Solar System (Fig. 2). Planet e is more compact and lies in a less populated region of the mass-radius diagram, resembling dense giant planets such as HATS-17 b (ref. ${ }^{16}$ ) and Kepler-539 b (ref. ${ }^{17}$ ). For the two smaller planets, $\mathrm{c}$ and $\mathrm{d}$, we calculated $3 \sigma$ upper limits on their masses of $0.24 M_{\text {Jup }}$ and $0.31 M_{\text {Jup }}$, respectively. The combination of the masses of all the pairs in the system are Hill-stable. Table 2 shows the final planetary parameters adopted for the system.

Core-accretion models of planetary evolution predict planets of $\sim 20 \mathrm{Myr}$ to be at the early stages of their contraction phase, showing very large radii and low densities ${ }^{1,18,19}$. Our results indicate that V1298 Tau b and e deviate from this picture. Figure 2 shows the position of the planets orbiting V1298 Tau in a mass-radius diagram compared to the known population of exoplanets. Similar to the case of AU Mic b (ref. ${ }^{7}$ ), the only other exoplanet of similar age with a mass measurement, the mass-radius relation of the planets orbiting V1298 Tau resembles that of the planets of our Solar System and of the general population of known transiting exoplanets. However, in contrast to the case of AU Mic b, the planets V1298 Tau b and e seem incompatible with the expected population derived from these models of evolution of planetary systems. Figure 3 shows the planets orbiting V1298 Tau with the expected population of exoplanets orbiting $1 M_{\odot}$ stars at the ages of $20 \mathrm{Myr}$ and $5 \mathrm{Gyr}$ (simulation NG76 (ref. ${ }^{20}$ ) using the Bern model ${ }^{1}$ ) and with the mass-radius tracks from other different models ${ }^{18,19}$. According to current theories these planets cannot reach this mass-radius configuration until hundreds of millions of years later. Considering their densities, it is not expected that the planets orbiting V1298 Tau will contract significantly in the future due to evaporation ${ }^{21}$. Our result suggests that some giant planets reach a mass-radius configuration compatible with the known mature population of exoplanets during their first $20 \pm 10 \mathrm{Myr}$ of age.

An alternative explanation to the characteristics of V1298 Tau $\mathrm{b}$ and e could be offered by an extreme enrichment in heavy elements compared to giant planets of the Solar System and the general population of transiting exoplanets ${ }^{22}$. Fractions of heavy elements of $40-60 \%$ and $60-80 \%$ of the mass of planets $b$ and e, respectively, would partially reconcile our results with the core-accretion evolutionary models $\mathrm{s}^{1,18,19}$. As the fraction of heavy elements of Jupiter is in the range of $2-8 \%$ (ref. ${ }^{23}$ ), this enrichment would correspond to 5-30 times the fraction of heavy elements of Jupiter for V1298 Tau $b$ and 7-40 times for V1298 Tau e. If we used the old planet configuration, planet $b$ could be explained with a fraction of heavy elements of 5-40\%, which is compatible with the expectation of the Bern model for Jupiter and Saturn ${ }^{1}$. Planet e would still need a fraction of $40-60 \%$ of heavy elements. Figure 3 shows the planets orbiting V1298 Tau with the mass-radius tracks derived for core-heavy exoplanets at the ages of $20 \pm 10$ Myr and the age of the Solar System. The models for core-heavy exoplanets predict that the planets will keep contracting, moving them to a region of the parameter space in which there are no known field exoplanets. If giant planets with such high metallicities existed and if this scenario were correct, it would suggest that the two planets formed farther away from their host star, possibly at separations of tens of astronomical units, consistent with those observed in protoplanetary disks imaged by the Atacama Large Millimeter/submillimeter Array, and that they experienced large-scale migration and accretion of planetesimals ${ }^{24}$. 
a
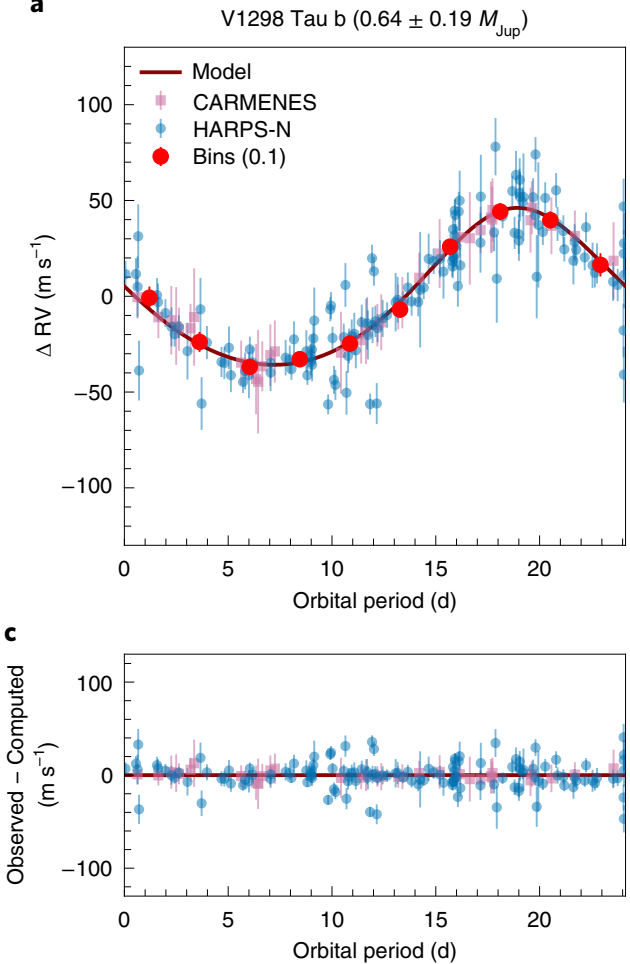

b

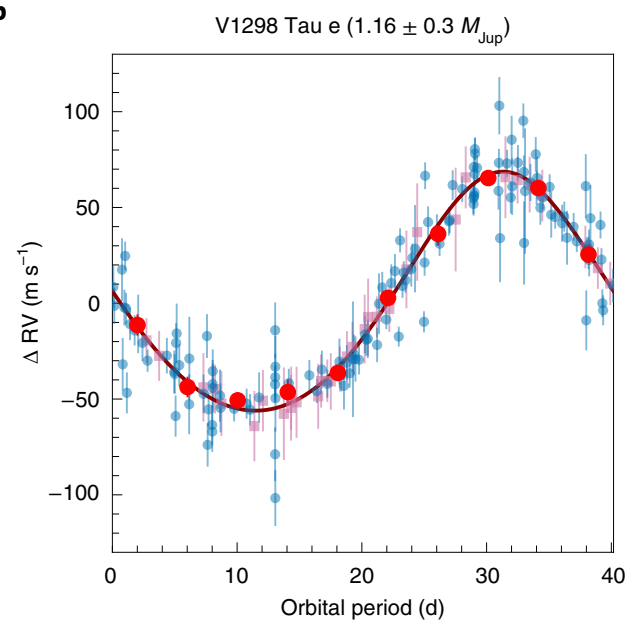

d

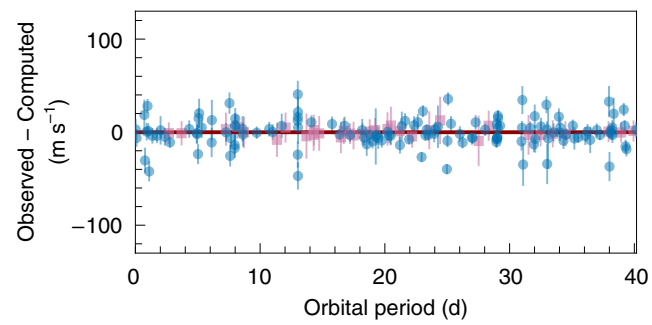

Fig. 1 | Phase-folded plots of the RV signals for the two planets of the V1298 Tau planetary system with significant mass measurements. a, Phase-folded representation of the best-fitting Keplerian orbit (red line) for V1298 Tau b. b, Same for V1298 Tau e. c,d, Residuals (observed minus computed) after the fit for both cases. For a better visualization, only HARPS and CARMENES data have been included. The large red dots show the data binned every $1 / 10$ th of the orbit. In all cases, $1 \sigma$ error bars (internal RV uncertainties) of the measurements are shown.

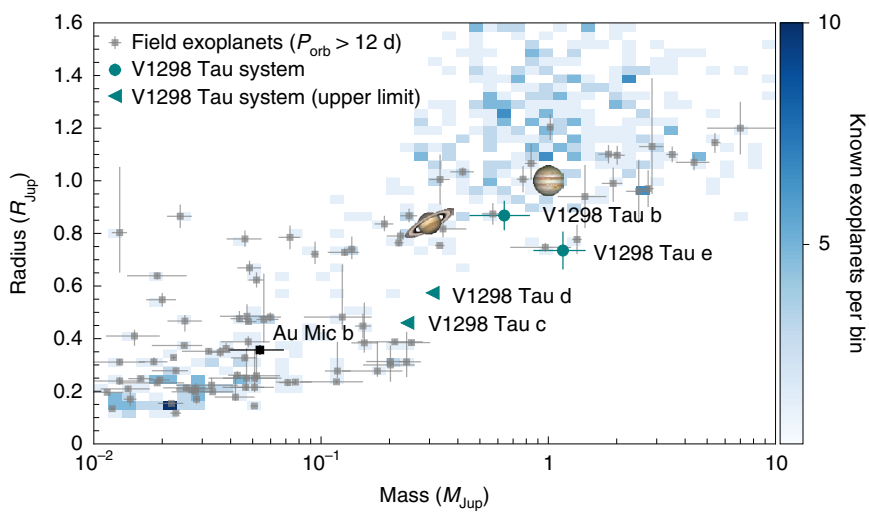

Fig. 2 | Planets of V1298 Tau in the context of the known planets. Histogram of the masses and radii of known planets for which the two parameters are determined with a precision better than $33 \%$. The planets orbiting V1298 Tau are highlighted in teal symbols, with their $1 \sigma$ error bars. The left-pointing arrows show the upper limits for the masses of V1298 Tau c and d. Jupiter and Saturn have been added for comparison. The black square shows the position of Au Mic b. The planets with orbital periods longer than 12 days are shown with dark grey symbols.

\section{Methods}

Data. HARPS-N and CARMENES RVs. HARPS- $\mathrm{N}^{25}$ is a fibre-fed high-resolution échelle spectrograph installed at the $3.6 \mathrm{~m}$ Telescopio Nazionale Galileo of the Roque de los Muchachos Observatory (La Palma, Spain). It has a resolving power of 115,000 over a spectral range of $360-690 \mathrm{~nm}$ and is contained in a temperatureand pressure-controlled vacuum vessel to avoid spectral drifts due to temperature and air pressure variations. It is equipped with its own pipeline, providing extracted and wavelength-calibrated spectra, as well as RV measurements and other data products, such as cross-correlation functions and the bisector of their line profiles. We obtained 132 observations between 2019 and 2020; 72 of those measurements were under Spanish time and the remaining 60 were in the context of the GAPS programme ${ }^{26,27}$, a long-term, multi-purpose, observational programme aimed at characterizing the global architectural properties of exoplanetary systems. On-source integration times were typically set to $900-1,200 \mathrm{~s}$. Using the HARPS-N data, we obtained the $\mathrm{S}_{\mathrm{MW}}$ index ${ }^{28}, \mathrm{H} \alpha$ index ${ }^{29}, \mathrm{Na}$ I index ${ }^{30}$ and $\mathrm{TiO}^{31}$ chromospheric indicators.

The CARMENES instrument ${ }^{32}$ consists of visual (VIS) and near-infrared (NIR) spectrographs covering $520-960 \mathrm{~nm}$ and $960-1,710 \mathrm{~nm}$ with a spectral resolution of 94,600 and 80,400 , respectively ${ }^{19}$. It is located at the $3.5 \mathrm{~m}$ Zeiss telescope at the Centro Astronómico Hispano-Alemán (Almería, Spain). We extracted the spectra with the CARACAL pipeline, based on flat-relative optimal extraction ${ }^{33}$. The wavelength calibration was performed by combining hollow cathode lamps (U-Ar, U-Ne and Th-Ne) and Fabry-Pérot etalons. The instrument drift during the nights is tracked with the Fabry-Pérot in the simultaneous calibration fibre. We obtained 35 observations between 2019 and 2020 .

RVs for HARPS-N and CARMENES were obtained using SERVAL. This software builds a high signal-to-noise template by co-adding all the existing observations, and then performs a maximum likelihood fit of each observed spectrum against the template, yielding a measure of the Doppler shift and its uncertainty. We obtained typical RV precisions of $9 \mathrm{~m} \mathrm{~s}^{-1}$ and $15 \mathrm{~m} \mathrm{~s}^{-1}$ for HARPS and CARMENES VIS measurements. For CARMENES NIR measurements we obtained a typical RV precision of $55 \mathrm{~m} \mathrm{~s}^{-1}$. We measured an r.m.s. of the RVs of $260 \mathrm{~m} \mathrm{~s}^{-1}, 197 \mathrm{~m} \mathrm{~s}^{-1}$ and $195 \mathrm{~m} \mathrm{~s}^{-1}$ for the HARPS-N, CARMENES VIS and CARMENES NIR, respectively. Contrary to what was expected, the CARMENES NIR RVs showed no significant reduction in dispersion compared to the VIS RVs. These NIR data show a large difference in precision compared to the visible channel and add no new temporal information. We opted to avoid increasing the complexity of the model and relied exclusively on the RVs coming from the visible channel.

HERMES RVs. The high-efficiency and high-resolution Mercator échelle spectrograph (HERMES) ${ }^{34}$ is installed at the $1.2 \mathrm{~m}$ Mercator telescope at the Roque de los Muchachos Observatory. The spectra were automatically processed by the HERMES pipeline, but later we derived our own RV measurements by cross-correlating the spectra with a K1 synthetic template ${ }^{35}$ taken from the HARPS-N reduction pipeline. This made the effective wavelength range used for the RV calculation similar to the HARPS-N wavelength range. We obtained 35 
Table 2 | Planetary parameters for the V1298 Tau system

\begin{tabular}{lllll} 
Parameter & V1298 Tau b & V1298 Tau c & V1298 Tau d & V1298 Tau e \\
\hline Orbital period $P_{\text {orb }}(\mathrm{d})$ & $24.1399 \pm 0.0015$ & $8.24892 \pm 0.00083$ & $12.4058 \pm 0.0018$ & $40.2 \pm 1.0$ \\
\hline Time of mid-transit $T_{0}(\mathrm{~d})$ & $7067.0486 \pm 0.0015$ & $7064.2801 \pm 0.0041$ & $7072.3907 \pm 0.0063$ & $7096.6226 \pm 0.0031$ \\
\hline Semi-major axis $a(\mathrm{au})$ & $0.1719 \pm 0.0027$ & $0.0841 \pm 0.0013$ & $0.1103 \pm 0.0017$ & $0.2409 \pm 0.0083$ \\
\hline Planet radius $R_{\mathrm{p}} / R_{*}$ & $0.0698 \pm 0.0024$ & $0.0371 \pm 0.0019$ & $0.0464 \pm 0.0020$ & $0.0583 \pm 0.0040$ \\
\hline Planet radius $R_{\mathrm{p}}\left(R_{\text {Jup }}\right)$ & $0.868 \pm 0.056$ & $0.460 \pm 0.034$ & $0.574 \pm 0.041$ & $0.735 \pm 0.072$ \\
\hline Incl. $\left(^{\circ}\right)$ & $>88.7$ & $>87.5$ & $>88.3$ & $>89.0$ \\
\hline RV semi-amplitude $K\left(\mathrm{~m} \mathrm{~s}^{-1}\right)$ & $41 \pm 12$ & $<22$ & $<25$ & $62 \pm 16$ \\
\hline Eccentricity & $0.134 \pm 0.075$ & $<0.30$ & $<0.20$ & $0.10 \pm 0.091$ \\
\hline Planet mass $\left(M_{\text {Jup }}\right)$ & $0.64 \pm 0.19$ & $<0.24$ & $<0.31$ & $1.16 \pm 0.30$ \\
\hline Density $\rho\left(\mathrm{g} \mathrm{cm}^{-3}\right)$ & $1.20 \pm 0.45$ & $<3.5$ & $<2.4$ & $3.6 \pm 1.6$ \\
\hline Par & & &
\end{tabular}

Parameters show $1 \sigma$ uncertainties. Upper and lower limits show the $99.7 \%$ confidence interval. $T_{0}$ is given in BJD -2450000 (where BJD is the barycentric Julian date).

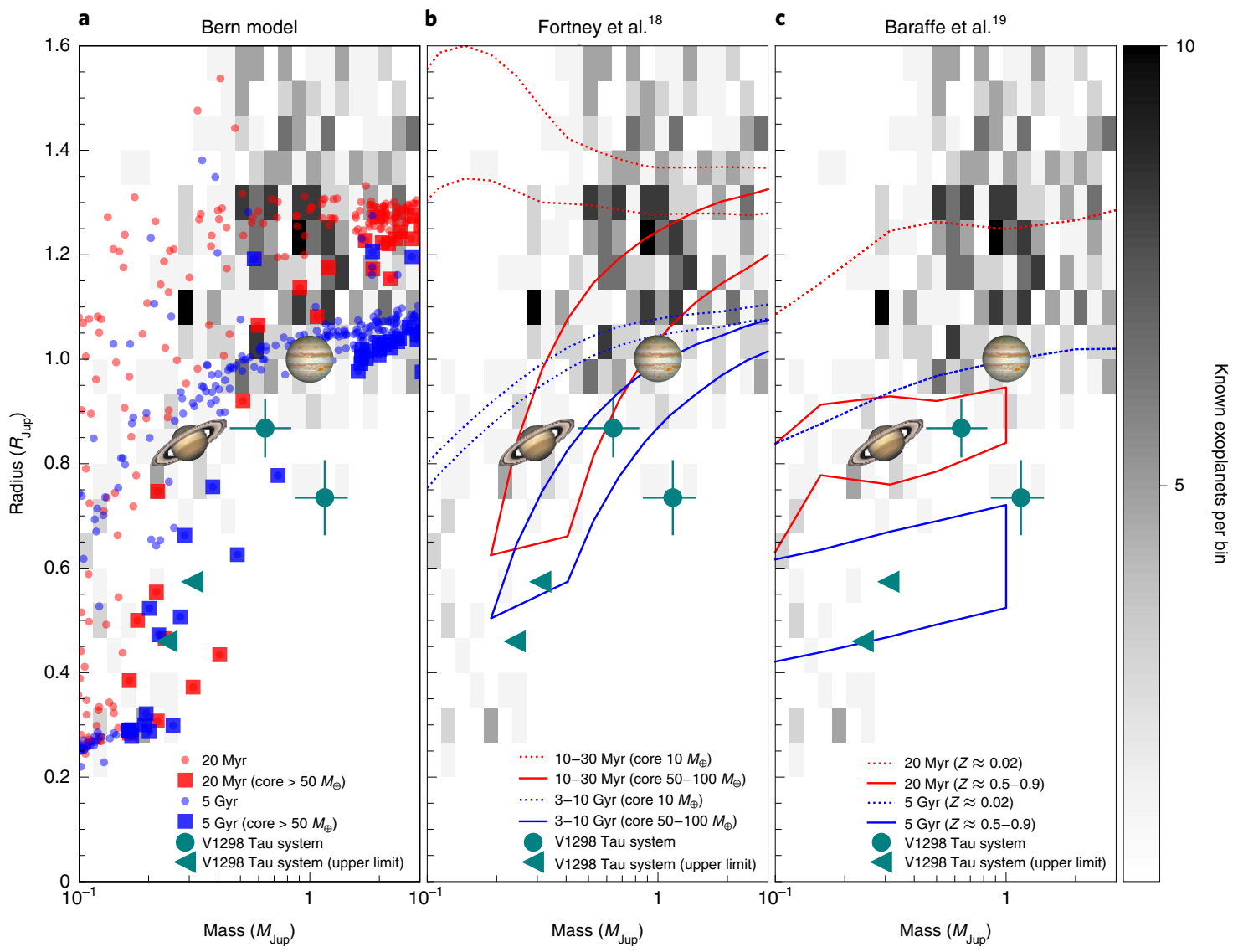

Fig. 3 | Planets of V1298 Tau in the context of the models of planetary evolution. a, Masses and radii of the planets orbiting V1298 Tau, with their $1 \sigma$ error bars, compared with the expected planetary population orbiting stars with solar mass and metallicity at 20 Myr and $5 \mathrm{Gyr}\left(\right.$ ref. ${ }^{20}$ ). Core-heavy planets have been highlighted. b. Masses and radii of the planets orbiting V1298 Tau compared with the mass-radius tracks from Fortney et al. ${ }^{18}$ for planets with light and heavy cores $\left(50-100 \mathrm{M}_{\oplus}\right)^{18}$. c. Masses and radii of the planets orbiting V1298 Tau compared with the mass-radius tracks from Baraffe et al. ${ }^{19}$ for planets with light and enriched cores $(Z \approx 0.5-0.9)$. The left-pointing arrows show the upper limits for the masses of $\mathrm{V} 1298$ Tau $\mathrm{c}$ and $\mathrm{d}$. All panels show the histogram of the known population of exoplanets and the positions of Jupiter and Saturn as reference points.

measurements during 18 individual winter nights in 2019-2020, with the goal of monitoring the shape of the activity variations. We obtained a typical RV precision of $55 \mathrm{~m} \mathrm{~s}^{-1}$ per observation and an r.m.s. of the RVs of $294 \mathrm{~m} \mathrm{~s}^{-1}$.

SES RVs. The STELLA échelle spectrograph (SES) ${ }^{36}$ is a high-resolution spectrograph installed at the $1.2 \mathrm{~m}$ STELLA telescope at the Teide Observatory (Tenerife, Spain). It has a resolving power of 55,000 over a wavelength range of $390-870 \mathrm{~nm}$ (ref. ${ }^{20}$ ). RVs were obtained by the automatic reduction pipeline, by cross-correlating the spectra with a synthetic stellar template ${ }^{35}$ with a temperature of $5,000 \mathrm{~K}$. We obtained 61 epochs spread across three months, during the winter of 2019-2020, with the goal of following the activity variations of V1298 Tau. We obtained a typical RV precision of $117 \mathrm{~m} \mathrm{~s}^{-1}$ per observation and an r.m.s. of the RVs of $309 \mathrm{~m} \mathrm{~s}^{-1}$.

LCOGT V-band photometry. We observed V1298 Tau with the $40 \mathrm{~cm}$ telescopes of the LCOGT ${ }^{25}$. These telescopes are equipped with a $3 \mathrm{k} \times 2 \mathrm{k}$ SBIG CCD camera with a pixel scale of $0.571 \mathrm{arcsec}$ providing a field of view of $29.2 \times 19.5 \mathrm{arcmin}$. 
We observed V1298 Tau in the $V$-band every $8 \mathrm{~h}$ over four months, during the winter of 2019-2020. The raw images were reduced by the BANZAI pipeline of the Las Cumbres Observatory, and aperture photometry was performed on the calibrated images using AstroImageJ v3.2.0 ${ }^{37}$. For each night, we selected a fixed circular aperture in AstroImageJ and performed aperture photometry on the target and on five reference stars of similar brightness. We obtained $250 \mathrm{~V}$-band measurements with a typical precision of $0.5 \%$ in relative flux.

K2 photometry. As complementary data to the spectroscopic dataset, we downloaded the available photometric light curve obtained by the Kepler Space Telescope K2 mission ${ }^{13}$ from the Mikulski Archive for Space Telescopes (MAST). This photometric dataset was taken in the long cadence mode, characterized by $30 \mathrm{~min}$ integration time. We adopted the EVEREST 2.0 (ref. ${ }^{38}$ ) light curve, which corrects for K2 systematics using a variant of the pixel-level decorrelation method ${ }^{39}$. This time series covers a time span of about $71 \mathrm{~d}$ (one Kepler quarter) from 8 February 2015 to 20 April 2015, which corresponds to the K2 Campaign 4.

FIES spectroscopy. We took a single exposure of 2,100 s of HD 284154 with the high-resolution $(R=67,000)$ fibre-fed échelle spectrograph (FIES) at the $2.6 \mathrm{~m}$ Nordic Optical Telescope of the Roque de los Muchachos Observatory. Observations were made on 18 August 2020.

Stellar parameters of V1298 Tau. Membership to Group 29. V1298 Tau is the low-mass companion of the warmer, G0-type star HD 284154 at a projected separation of $97.7 \mathrm{arcsec}$ (or 10,600 au at the distance of the system). The pair belongs to the recently identified Group 29 (ref. ${ }^{10}$ ), which is a young, sparse association of coeval stars in the Taurus region, all of which share very similar proper motions and distances according to the Tycho-Gaia astrometric catalogue (TGAS) $^{40,41}$. Using updated trigonometric parallaxes and other astrometric determinations provided by the Gaia Data Release 2 (ref. ${ }^{26}$ ), we confirm that both V1298 Tau and HD 284154 are proper motion companions located at a distance that is compatible with that of the Group (Table 1). The Galactic velocities $U, V$ and $W$ of V1298 Tau and HD 284154 completely overlap with the distribution of the space motions of Group 29 members, thus providing additional support for the membership of V1298 Tau and HD 284154 in this association.

Effective temperature, surface gravity and metallicity of V1298 Tau. We derived the stellar parameters and metallicity of V1298 Tau using the high-resolution HARPS-N spectra. The extracted, blaze-corrected, two-dimensional spectra were corrected for barycentric velocity (varying from $+10.8 \mathrm{~km} \mathrm{~s}^{-1}$ to $-30.4 \mathrm{~km} \mathrm{~s}^{-1}$ ) and for RV (varying from $+14.4 \mathrm{~km} \mathrm{~s}^{-1}$ to $+15.7 \mathrm{~km} \mathrm{~s}^{-1}$ ), and normalized to unity order by order with a third-degree polynomial using our own IDL-based automated code $\mathrm{e}^{42}$. All orders of all spectra were co-added and merged with a wavelength step of $0.01 \AA$ per pixel. The resulting one-dimensional spectrum shows a signal-to-noise ratio of $\sim 107,222,284,412$ and 391 at $4,200 \AA, 4,800 \AA, 5,400 \AA$, $6,000 \AA$ and $6,600 \AA$, respectively.

Using the same automated code, we normalized and combined the HARPS-N spectra from our RoPES RV program ${ }^{43}$ of three other stars (HD 220256, HD 20165 and $\epsilon$ Eri) with similar spectral types to be used as comparison stars. We compared the HARPS-N spectra of these templates with that of V1298 Tau to derive the projected rotation velocity of V1298 Tau. To reduce the computing load we restricted the calculation to the spectral range of 5,355-5,525 and performed a Markov chain Monte Carlo simulation with 5,000 chains implemented in emcee v3.0.0 $0^{44}$. The mean stellar projected rotation velocity of V1298 Tau obtained from the three templates is $23.8 \pm 0.5 \mathrm{~km} \mathrm{~s}^{-1}$, which is consistent with the measurement derived from the stellar radius and rotation period $\left(22.2 \pm 1.3 \mathrm{~km} \mathrm{~s}^{-1}\right)$.

To estimate the stellar parameters of V1298 Tau ( $T_{\text {eff }}$ and $\log g$, and metallicity, $[\mathrm{Fe} / \mathrm{H}] ; A(\mathrm{X})=\log [N(\mathrm{X}) / N(\mathrm{H})]+12$, with $N(\mathrm{X})$ the number density of element $\mathrm{X}$, and $[\mathrm{Fe} / \mathrm{H}]=A(\mathrm{X})-A_{\odot}(\mathrm{X})$, with $\left.\mathrm{X}=\mathrm{Fe}\right)$, we used three different codes, which allowed us to check the consistency of the results. First, we used the FERRE $\operatorname{code}^{45}$ with a grid of synthetic spectra ${ }^{46}$ with a microturbulence velocity fixed at $\xi_{\text {mic }}=1.5 \mathrm{~km} \mathrm{~s}^{-1}$ to fit the HARPS-N spectrum of V1298 Tau, providing $T_{\text {eff }} / \log g / A(\mathrm{Fe})=5,010 / 4.48 / 7.20$ (note that the canonical solar Fe abundance is $A_{\odot}(\mathrm{Fe})=7.50\left(\right.$ ref. $\left.{ }^{47}\right)$ ). For comparison, we also analysed the HARPS-N spectrum of the star $\epsilon$ Eri and the Kurucz solar ATLAS spectrum ${ }^{48}$, both broadened with a rotation profile of $24 \mathrm{~km} \mathrm{~s}^{-1}$, and obtained $T_{\text {eff }} / \log g / A(\mathrm{Fe})=5,085 / 4.91 / 7.24$ and $5,912 / 4.74 / 7.31$, respectively. FERRE uses a running mean filter to normalize both synthetic and observed spectra and fits a wide spectral range of the HARPS-N spectrum (4,500-6,800 $)$, masking out the Balmer and Na I D lines, which for the young V1298 Tau star could show their cores in emission. Taking the analysis of the Solar ATLAS as the solar reference, this first method gives $[\mathrm{Fe} / \mathrm{H}]=-0.11$ as the metallicity of V1298 Tau. We suspect that the slightly low metallicity is related to the relatively high microturbulence adopted for the grid of synthetic spectra ${ }^{46}$. The expected microturbulence velocity should be $\xi_{\text {mic }}=0.85 \mathrm{~km} \mathrm{~s}^{-1}$ (ref. ${ }^{49}$ ), so this may be the reason for the metallicity derived with this method appearing to be slightly lower than the solar value.
Second, we used the SteParSyn code (Tabernero et al., manuscript in preparation), a Bayesian code that uses a synthetic grid of small spectral regions of $3 \AA$ around $95 \mathrm{Fe}$ lines with a fixed $\xi_{\text {mic }}=0.85 \mathrm{~km} \mathrm{~s}^{-1}$. The results of this second method are 5,041/4.24/7.62 and a metallicity $[\mathrm{Fe} / \mathrm{H}]=+0.16$.

Third, we implemented a Bayesian python code that compares the observed spectrum with a synthetic spectrum in the spectral range 5,350-5,850 $\AA$ (Extended Data Fig. 1). We performed a Markov chain Monte Carlo simulation with 5,000 chains implemented in emcee ${ }^{44}$. We used a small $3 \times 3 \times 3$ grid of synthetic spectra with $T_{\text {eff }} / \log g / A(\mathrm{Fe})$ values of $4,750-5,250 / 3.5-4.5 / 7.0-8.0$ and with steps of $250 \mathrm{~K} / 0.5 \mathrm{dex} / 0.5 \mathrm{dex}$, computed with the SYNPLE code, assuming a microturbulence $\xi_{\text {mic }}=0.85 \mathrm{~km} \mathrm{~s}^{-1}$; we also used ATLAS9 model atmospheres with solar $\alpha$-element abundances ${ }^{50}([\alpha / \mathrm{Fe}]=0)$ and the same line list as in first method. This third method delivered $T_{\text {eff }} / \log g / A(\mathrm{Fe})=5,071 / 4.25 / 7.44$ and a metallicity $[\mathrm{Fe} / \mathrm{H}]=+0.07$ for $\mathrm{V} 1298 \mathrm{Tau}$ (the result for the broadened solar ATLAS is $5,753 / 4.48 / 7.37)$.

Taking into account the slightly different results from the three different methods, we adopted the values $T_{\text {eff }} / \log g /[\mathrm{Fe} / \mathrm{H}]=5,050 \pm 100 / 4.25 \pm 0.20 /$ $+0.10 \pm 0.15$ for V1298 Tau. Finally, we checked the derived effective temperature by applying the implementation of the infrared flux method (IRFM) ${ }^{51}$. Using the available photometry in the infrared bands $J H K_{S}$ from the Two Micron All-Sky Survey (2MASS) ${ }^{30}$ and the Johnson $V$ magnitude from the AAVSO Photometric All-Sky Survey (APASS $)^{52}$, and adopting $E(B-V)=0.061$ from the dust maps ${ }^{53}$ corrected $^{54}$ using the distance of $108 \mathrm{pc}$ to V1298 $\mathrm{Tau}^{40}$, we applied the IRFM to obtain $_{\text {a TIRFM }}=5,047 \pm 66 \mathrm{~K}$, in agreement with the spectroscopic value. Assuming an extinction $E(B-V)=0.024 \pm 0.015$ (ref. ${ }^{11}$ ), we obtained $T_{\mathrm{IRFM}}=4,947 \pm 67 \mathrm{~K}$, and $4,928 \pm 67 \mathrm{~K}$ for $E(B-V)=0$.

Effective temperature, surface gravity and metallicity of HD 284154. HD 284154 is resolved as a double-lined spectroscopic binary, which has been identified as a wide binary of V1298 $\mathrm{Tau}^{41}$. Using the FIES spectrum, we estimated an RV difference of $\delta \mathrm{RV}=43.6 \pm 1.0 \mathrm{~km} \mathrm{~s}^{-1}$ between the two stellar components, and we derived an identical stellar rotation of $V_{\text {rot }}=10 \pm 1 \mathrm{~km} \mathrm{~s}^{-1}$ from the double-peaked cross-correlation function of the observed FIES spectrum cross-correlated with a mask of isolated and relatively strong Fe lines. Assuming that both binary components have the same mass (see below), the centre-of-mass RV is $+14.8 \pm 0.7 \mathrm{~km} \mathrm{~s}^{-1}$, which is perfectly compatible with the centre-of-mass RV of V1298 Tau.

We applied the third method to derive the stellar parameters and metallicity of both stellar components of HD 284154. We assumed that both stars have the same luminosity and origin, and therefore the same stellar mass and metallicity. We then generated a grid of synthetic spectra in the $T_{\text {eff }} / \log g / A(\mathrm{Fe})$ parameter range of 5,500-6,000/3.5-4.5/7.0-8.0 and with steps of $250 \mathrm{~K} / 0.5 \mathrm{dex} / 0.5 \mathrm{dex}$, with a fixed $\xi_{\text {mic }}=0.95 \mathrm{~km} \mathrm{~s}^{-1}$, and added the fluxes of each component separated by $\delta R V=43.6 \mathrm{~km} \mathrm{~s}^{-1}$. We obtained $T_{\text {eff }} / \log g /$ $[\mathrm{Fe} / \mathrm{H}]=5,700 \pm 100 / 4.35 \pm 0.20 /+0.05 \pm 0.15$ for HD 284154 .

Lithium abundance. We used the MOOG code $^{55}$ and ATLAS9 model atmospheres to derive the lithium abundances of V1298 Tau and HD 284154 (Extended Data Fig. 2), with the approximation of local thermodynamic equilibrium (LTE). We applied the non-LTE corrections ${ }^{56}$ to get a $\mathrm{Li}$ abundance of $A(\mathrm{Li})=3.43 \pm 0.15$ and $3.24 \pm 0.15$ for V1298 Tau and HD 284154, respectively, roughly consistent with the solar meteoritic value ${ }^{47}$.

Masses, radii and luminosities. We determined the bolometric luminosity of both V1298 Tau and HD 284154 by transforming the observed magnitudes into bolometric magnitudes using Gaia distances and colour-bolometric corrections $\mathrm{s}^{57}$. We confirmed that the obtained values (Table 1) are fully compatible at the $1 \sigma$ level with those derived from the integration of the photometric spectral energy distributions using the Virtual Observatory Spectral Energy Distribution Analyzer $^{58}$ (VOSA) tool for the stellar effective temperatures. The radius of each star was then obtained from the Stefan-Boltzmann equation; we split the luminosity of HD 284154 into two identical parts to account for its nearly equal-mass binary nature and arbitrarily augmented the error in the luminosity determination of each component by a factor of two. Masses were obtained from the comparison of the derived effective temperatures and bolometric luminosities with various stellar evolutionary models available in the literature ${ }^{59-61}$. All models are consistent within the error bars. Uncertainties in the mass determination account for the temperature and luminosity uncertainties and also for the dispersion of the results from the different models including models with slightly different metallic composition. We obtain mass and radius estimates of $1.170 \pm 0.060 M_{\odot}$ and $1.278 \pm 0.070 M_{\odot}$ for V1298 Tau, and $1.28 \pm 0.06 R_{\odot}$ and $1.477 \pm 0.082 R_{\odot}$ for each of the stars of HD 284154. All values are provided in Table 1 . In addition, we inferred the stellar parameters for V1298 Tau and HD 284154 from stellar evolution models using a Bayesian inference method ${ }^{62}$. This Bayesian analysis makes use of the PARSEC v1.2S library of stellar evolution models ${ }^{61}$. It takes the absolute $G$ magnitude (using the parallax) and the colour $G_{\mathrm{BP}}-G_{\mathrm{RP}}$ from Gaia Data Release 2 (ref. ${ }^{40}$ ), and assumes solar metallicity, with $[\mathrm{Fe} / \mathrm{H}]=0.00 \pm 0.20$, returning theoretical predictions for other stellar parameters. For HD 284154 it was assumed that this binary (whose Gaia photometry was 
corrected by adding $0.7526 \mathrm{mag}$ ) is in the pre-main-sequence phase due to the expected youth of the association. For V1298 Tau we obtained a $\log L$ of $-0.040 \pm 0.009$, an effective temperature of $4,929 \pm 32 \mathrm{~K}$, a mass of $1.17 \pm 0.03 M_{\odot}$, a radius of $1.310 \pm 0.027 R_{\odot}$ and a $\log g$ of $4.271 \pm 0.028$. For HD 284154 we obtained a $\log L$ of $0.299 \pm 0.009$, an effective temperature of $5,699 \pm 55 \mathrm{~K}$, a mass of $1.263 \pm 0.013 M_{\odot}$, a radius of $1.45 \pm 0.04 R_{\odot}$ and a $\log g$ of $4.218 \pm 0.020$.

Age estimation. Extended Data Fig. 3 shows that the photometric sequence of Group 29 is compatible with the isochrones of 10-30 Myr. This photometric sequence of Group 29 is sub-luminous compared to that of the Upper Scorpius association, and is very similar to that of the Beta Pictoris moving group. This suggests that the Group 29, and hence the V1298 Tau system, is older than the Upper Scorpius association $\left(5-11 \mathrm{Myr}\left(\right.\right.$ ref. $\left.{ }^{10}\right)$ ) and has an age similar to that of Beta Pictoris $\left(20 \pm 10 \mathrm{Myr}\left(\right.\right.$ ref. $\left.{ }^{63}\right)$ ). The rotation period of V1298 Tau $\left(2.865 \pm 0.012 \mathrm{~d}^{\left.\left(\text {ref. }^{9}\right)\right)}\right.$ points in a similar direction. It fits perfectly with stars with similar spectral types of very young associations such as Rho Ophiuchus, Taurus, Upper Scorpius and the Taurus foreground population ${ }^{64}$, with ages in the range of 1-30 Myr, but rotates faster than stars of similar spectral types in open clusters such as the Pleiades $\left(\sim 110 \mathrm{Myr}\left(\right.\right.$ ref. $\left.\left.{ }^{65}\right)\right)$ or Praesepe $\left(600-800 \mathrm{Myr}\left(\right.\right.$ ref. $\left.{ }^{66}\right)$ ). The lithium content of V1298 Tau also allows us to constrain its age, as this element is destroyed in low-mass stars on timescales of tens of millions of years. Comparing the equivalent width of the lithium line of V1298 Tau ( $400 \mathrm{~m} \AA)$ with that of stars in open clusters and young moving groups of different ages ${ }^{67}$, we can conclude that the lithium content of V1298 Tau is compatible with an age of $1-20 \mathrm{Myr}$, and is larger than in stars in open clusters such as IC 2391 and IC 2602, with estimated ages of 35-50 Myr (ref. ${ }^{68}$ ). V1298 Tau exhibits an X-ray emission of $4.58_{-1.44}^{+1.71} \times 10^{30} \mathrm{erg} \mathrm{s}^{-1}$ (ref. ${ }^{69}$ ), compatible with an activity stronger than that of the stars in the Pleiades ${ }^{70,71}$, and has an ultraviolet excess $(8.15 \pm 0.05$ magnitude difference between the magnitude in the near-ultraviolet and the magnitude in the J band) characteristic of stars younger than $\sim 100 \mathrm{Myr}\left(\right.$ ref. ${ }^{72}$ ). In addition, employing the same Bayesian inference method used in the previous section ${ }^{62}$, we also obtained estimates for the ages of V1298 Tau and HD 284154 of $9 \pm 2$ Myr and $13 \pm 4 \mathrm{Myr}$, respectively. Using all the previous results, we can constraint the age of V1298 Tau to be $20 \pm 10$ Myr.

Modelling. We fitted the K2 photometry, LCOGT photometry and RV time series simultaneously, and modelled the activity signals in RV and the LCOGT photometry using Gaussian processes (GP) with celerite v0.4.0 $0^{73}$. We used a variation of the quasi-periodic kernel described in equation (56) of the original celerite article, with the explicit addition of a second mode at half the rotation period (PQP2 here):

$$
\begin{aligned}
k(\tau)= & \frac{A^{2}}{2+C}\left[\mathrm{e}^{-\tau / L_{1}}\left(\cos \left(\frac{2 \pi \tau}{P_{\text {rot }}}\right)+(1+C)\right)+\Delta^{2} \mathrm{e}^{-\tau / L_{2}}\left(\cos \left(\frac{4 \pi \tau}{P_{\text {rot }}}\right)\right.\right. \\
& +(1+C))]+\left(\sigma^{2}(t)+\sigma_{j}^{2}\right) \delta_{\tau},
\end{aligned}
$$

where $A$ represents the covariance amplitude, $P_{\text {rot }}$ is the rotation period, $L_{1}$ and $L_{2}$ represent the timescale of the coherence of the periodicity at the rotation period and its first harmonic, $\Delta$ represents the scaling in amplitude of the variability at the first harmonic of the rotation period and $C$ represents the balance between the periodic and the non-periodic components. The equation also includes a term of uncorrelated noise $(\sigma)$ that is independent for every instrument and added quadratically to the diagonal of the covariance matrix to account for all unmodelled noise components, such as uncorrected activity or instrumental instabilities. $\delta_{\tau}$ is the Kronecker delta function and $\tau$ represents an interval between two measurements, $t-t^{\prime}$. This kernel behaves similarly to the classical quasi-periodic kernel ${ }^{74}$. The base version of this celerite kernel was successfully used to model the variations of Proxima Centauri to the level of the instrumental precision ${ }^{75}$. To model the activity variations in the $\mathrm{K} 2$ photometry, we used a combination of two simple harmonic oscillators (SHOs) centred at the rotation period of the star and its first harmonic. This kernel has been shown to appropriately model the photometric variations of V1298 Tau'. The SHO kernel is described in equation (2). To better constrain the behaviour of the GP in its description of the activity-induced RV variations, some of the hyper-parameters are shared between the GP of the LCOGT photometry and the $\mathrm{RV}^{26}$. The period and timescales of coherence of the variability are shared parameters, whereas the amplitudes and mix factors are independent. As activity signals are known to have a chromatic dependence ${ }^{15,76}$, we split the dataset by instruments and gave independent amplitudes of the activity signals to each instrument. The analysis considered a zero-point value and a noise term (jitter) for each dataset as free parameters to be optimized simultaneously, with the exception of the K2 data. For the K2 data we opted to manually include the white-noise component given in the discovery paper ${ }^{15}$. The K2 observations were obtained in 2017, whereas the LCOGT and RV data were obtained during 2019 and 2020. As the activity is not expected to remain stable after such a long time, we used two groups of hyper-parameters for the two different observing campaigns. We measure the final planetary parameters by fitting transits of the K2 light curve using the PyTransit v2.0 package ${ }^{77}$ with quadratic limb darkening ${ }^{78}$ and with Keplerian orbits implemented with RadVel v1.4.0 $0^{79}$ in the RVs.

$$
\begin{aligned}
k(\tau)= & A^{2} \mathrm{e}^{-\tau / L}\left\{\begin{array}{c}
\cosh \left(\nu 2 \pi \tau / P_{\text {rot }}\right)+\frac{P_{\text {rot }}}{2 \pi \nu L} \sinh \left(\nu 2 \pi \tau / P_{\text {rot }}\right), P_{\text {rot }}>2 \pi L \\
2\left(1+\frac{2 \pi \tau}{P_{\text {rot }}}\right), P_{\text {rot }}=2 \pi L \\
\cos \left(\nu 2 \pi \tau / P_{\text {rot }}\right)+\frac{P_{\text {rot }}}{2 \pi \nu L} \sin \left(\nu 2 \pi \tau / P_{\text {rot }}\right), P_{\text {rot }}<2 \pi L
\end{array}\right\} \\
& +\left(\sigma^{2}(t)+\sigma_{j}^{2}\right) \delta_{\tau},
\end{aligned}
$$

with $\nu=\left(1-\left(2 L / P_{\text {rot }}\right)^{-2}\right)^{1 / 2}$

To sample the posterior distribution and obtain the Bayesian evidence of the model (that is, marginal likelihood, $\ln Z$ ) we relied on nested sampling ${ }^{80}$ using dynesty v1.1 $1^{81}$. We initialized a number of live points equal to $N(N+1) / 2$, with $N$ being the number of free parameters.

We detected the signals corresponding to planets $b$ and e (Fig. 1) and derived upper limits for the amplitudes of the signals corresponding to planets $\mathrm{c}$ and $\mathrm{d}$ (Extended Data Fig. 4). This was our most significant model, with a measured $\ln Z$ of $-4,472$. To confirm our results we repeated the analysis described above using the combination of two SHOs to model the RV and LCOGT variations. We obtained a similar result, with larger amplitude for planet $b$ and smaller uncertainties but a larger r.m.s. of the residuals. This model proved to be less significant ( $\ln Z$ of $-4,549)$. We also attempted to confirm the results using the quasi-periodic GP kernel to model the activity variations in the RV and LCOGT data, implemented using George v0.3.1 ${ }^{82}$ (equation (3)). Previous researchers have found it effective to study young stars ${ }^{83}$. In this case we obtain lower amplitudes for the signals attributed to planets $\mathrm{b}$ and $\mathrm{e}$, and higher amplitudes for planet $\mathrm{c}$. This was the least favoured of the models we tested ( $\ln Z$ of $-4,563)$. For the most favoured model (PQP2) we tested the difference between having four planetary components in the RV, two planetary components (b and e) and no planetary components. We found that a model with two Keplerian components in the RV is much more likely than a model with no planetary signals, with a $\Delta \ln Z>25$ (false alarm probability $<0.1 \%$ ), and also more much likely than a model with only planet b, with a $\Delta \ln Z>20$ (false alarm probability $<0.1 \%$ ). The model with four Keplerian components is less significant than the model with two Keplerian components, which is not surprising considering we could not detect the RV signals of planets $\mathrm{c}$ and $\mathrm{d}$.

$$
k(\tau)=A^{2} \exp \left[-\frac{\tau^{2}}{L}-\frac{\sin ^{2}\left(\pi \tau / P_{\mathrm{rot}}\right)}{2 \omega^{2}}\right]+\left(\sigma^{2}(t)+\sigma_{j}^{2}\right) \delta_{\tau} .
$$

As the results coming from the different GP models are slightly different, we performed simulations to test the accuracy of the amplitude measurements in this particular case. To do that we subtracted the detected planetary signals from the RV to create an 'activity only' dataset. Following the same procedure as with our original RV dataset, we tested that all the models recovered amplitudes that are consistent with zero as the periods of the planets. Later we injected planetary signals at different amplitudes to study the behaviour of every model. The results were very similar to what we had already found. The PQP2 kernel recovered the amplitudes of the signals corresponding to planets $\mathrm{b}$ and e within a $5 \%$ accuracy for amplitudes larger than $20 \mathrm{~m} \mathrm{~s}^{-1}$. The model showed a tendency to underestimate the amplitudes of planets $\mathrm{c}$ and $\mathrm{d}$ by a $20 \%$ margin for amplitudes smaller than $20 \mathrm{~m} \mathrm{~s}^{-1}$. The combination of two SHO kernels consistently overestimated the amplitude of planet $b$, while underestimating the amplitudes of the three remaining signals. The model using this kernel also recovered smaller uncertainties for the Keplerian amplitudes in all scenarios. The quasi-periodic kernel accurately recovered the amplitudes of planets $\mathrm{c}$ and $\mathrm{d}$, and it strongly underestimated the amplitudes of the signals corresponding to planets b and e, sometimes by a $50 \%$ margin. This is not a fully unexpected behaviour, as the more flexible GP kernels have a higher rate of false negatives ${ }^{76}$. Extended Data Fig. 5 shows the comparison between the injected and recovered planetary amplitudes using the three GP kernels. The PQP2 kernel provided the most consistent results for all the tested combinations.

To further test our results we opted for a different approach based on the correlation of the RVs with the photometric data. In spot-dominated stars, the activity-induced RV variations are correlated with the gradient of the flux ${ }^{76}$. This correlation can be used to detrend the data from stellar activity. As we have contemporaneous, but not simultaneous, photometry, we calculated the gradient from the model of the photometry. We modelled the rotation using a third-order polynomial against the derivative of the flux. A first attempt left some residual power at the first harmonic of the rotation period, which led us to include a sinusoidal component at that period. Our activity model is then defined as

$$
\begin{aligned}
\mathrm{RV}_{\text {rot }}= & \mathrm{C} 1 \frac{\mathrm{dFlux}}{\mathrm{d} t}+\mathrm{C} 2\left(\frac{\mathrm{dFlux}}{\mathrm{d} t}\right)^{2}+\mathrm{C} 3\left(\frac{\mathrm{dFlux}}{\mathrm{d} t}\right)^{3} \\
& +A_{\text {rot }} \sin \left(4 \pi \frac{(t-\mathrm{T} 0)}{P_{\text {rot }}}\right),
\end{aligned}
$$

where $\mathrm{T} 0$ was parametrized as $\mathrm{JD}_{0}+P_{\text {rot }} \phi$, with $\mathrm{JD}_{0}=2458791.627$ and $\phi$ as the phase.

Using this model we recovered a solution very similar to that with the mixture of two SHO kernels, although with much larger residuals. We detected the presence of the planets V1298 Tau b and e, and measured upper limits for the amplitudes of 
planets $\mathrm{c}$ and $\mathrm{d}$. We measure the amplitude of planet e to be much larger than that of the GP models, which might be caused by the Keplerian model absorbing some unmodelled activity.

Supplementary Table 1 shows the parameters used in the fit, the datasets involved in fitting every parameter, the priors and the results obtained for the different models tested. Extended Data Fig. 6 shows the corner plot of the parameters of the most significant model. Extended Data Fig. 7 shows the RV with the best-fit model for the raw data, activity-filtered data, planet-filtered data and residuals, along with the GLS periodogram ${ }^{84}$ of each of those datasets. To represent the activity model we used a weighted average of the models for the different instruments. Extended Data Fig. 8 shows the activity component for every individual instrument during the most intense phase of the campaign. Extended Data Fig. 9 shows the best fit to the contemporaneous photometry and Extended Data Fig. 10 shows the best fit to the K2 observations.

Lessons learned and limitations. We found that the signal phase-folded to the rotation period shows clearly the two modes of oscillation that our favoured GP kernel describes. The amplitude of the rotation signal is eight times larger than the amplitude measured for the signal related to V1298 Tau b, and five times larger than the signal related to planet e. In the context of young exoplanets, the stellar activity signals engulf those signals related to the planets and therefore similarly large observational efforts with precise RV measurements will be required.

We found that not all GP kernels behaved the same at all timescales in our dataset. The classic quasi-periodic kernel handles short-period signals quite well. However for longer period signals it seems to absorb a significant part of the Keplerian components, causing a clear underestimation of the measured amplitudes. The mixture of SHO kernels had the opposite behaviour. It underfits the activity component, leaving larger residuals and causing an overestimation of (some of) the Keplerian amplitudes. We found that our kernel of choice (PQP2) provides a better description of the activity variations of V1298 Tau and a more accurate determination of the Keplerian amplitudes.

It is important to remain cautious about the mass determined for the planet V1298 Tau e. The original detection did not constrain the orbital period, which is derived purely from the RV information. We studied the $\mathrm{S}_{\mathrm{MW}}$ index ${ }^{28}, \mathrm{H} \alpha$ index ${ }^{29}, \mathrm{Na} \mathrm{I}$ index ${ }^{30}$ and $\mathrm{TiO}^{31}$ chromospheric indicators following a procedure very similar to that with the RV data, and did not find any significant periodicity (aside from the rotation) at periods shorter than 150 days, which favours the planetary hypothesis. However, disentangling planetary signals from stellar activity in RV in young stars such as V1298 Tau is a very challenging task. Without the confirmation of its orbital period by transit photometry, it is very difficult to completely exclude a stellar origin (or contribution) from the signal.

\section{Data availability}

The RV, LCOGT and K2 time series are available at https://cloud.iac.es/index. $\mathrm{php} / \mathrm{s} /$ jto2dxfHRF2Aw2B. The public high-resolution spectroscopic raw data used in the study can be freely downloaded from the corresponding facility archives. Proprietary raw data are available from the corresponding author upon reasonable request. Source data are provided with this paper.

\section{Code availability}

The SERVAL template-matching radial velocity measurement tool, celerite, George, emcee, dynesty, RadVel, PyTransit, AstroImageJ, SYNPLE, StePar, FERRE and MOOG are easily accessible open-source projects. Additional software is available upon request.

Received: 23 August 2021; Accepted: 4 October 2021; Published online: 02 December 2021

\section{References}

1. Mordasini, C. et al. Characterization of exoplanets from their formation. II. The planetary mass-radius relationship. Astron. Astrophys. 547, A112 (2012)

2. D’Angelo, G., Weidenschilling, S. J., Lissauer, J. J. \& Bodenheimer, P. Growth of Jupiter: formation in disks of gas and solids and evolution to the present epoch. Icarus 355, 114087 (2021).

3. Donati, J. F. et al. A hot Jupiter orbiting a 2-million-year-old solar-mass T Tauri star. Nature 534, 662-666 (2016).

4. Damasso, M. et al. The GAPS Programme at TNG. XXVII. Reassessment of a young planetary system with HARPS-N: is the hot Jupiter V830 Tau b really there? Astron. Astrophys. 642, A133 (2020).

5. David, T. J., Hillenbrand, L. A., Cody, A. M., Carpenter, J. M. \& Howard, A. W. K2 discovery of young eclipsing binaries in Upper Scorpius: direct mass and radius determinations for the lowest mass stars and initial characterization of an eclipsing brown dwarf binary. Astrophys. J. 816, 21 (2016).

6. Plavchan, P. et al. A planet within the debris disk around the pre-main-sequence star AU Microscopii. Nature 582, 497-500 (2020).
7. Klein, B. et al. Investigating the young AU Mic system with SPIRou: large-scale stellar magnetic field and close-in planet mass. Mon. Not. R. Astron. Soc. 502, 188-205 (2021).

8. David, T. J. et al. A warm Jupiter-sized planet transiting the pre-main-sequence star V1298 Tau. Astron. J. 158, 79 (2019).

9. David, T. J. et al. Four newborn planets transiting the young solar analog V1298 Tau. Astrophys. J. Lett. 885, L12 (2019).

10. Oh, S., Price-Whelan, A. M., Hogg, D. W., Morton, T. D. \& Spergel, D. N. Comoving stars in Gaia DR1: an abundance of very wide separation comoving pairs. Astron. J. 153, 257 (2017).

11. Howell, S. B. et al. The K2 mission: characterization and early results. Publ. Astron. Soc. Pac. 126, 398 (2014)

12. Beichman, C. et al. A mass limit for the young transiting planet V1298 Tau b. Res. Notes AAS 3, 89 (2019).

13. Brown, T. M. et al. Las Cumbres Observatory Global Telescope network. Publ. Astron. Soc. Pac. 125, 1031 (2013).

14. Rasmussen, C. E. \& Williams, C. K. I. Gaussian Processes for Machine Learning (MIT Press, 2006).

15. David, T. J. et al. Age determination in Upper Scorpius with eclipsing binaries. Astrophys. J. 872, 161 (2019).

16. Brahm, R. et al. HATS-17b: a transiting compact warm Jupiter in a 16.3 day circular orbit. Astron. J. 151, 89 (2016).

17. Mancini, L. et al. Kepler-539: a young extrasolar system with two giant planets on wide orbits and in gravitational interaction. Astron. Astrophys. 590, A112 (2016).

18. Fortney, J. J., Marley, M. S. \& Barnes, J. W. Planetary radii across five orders of magnitude in mass and stellar insolation: application to transits. Astrophys J. 659, 1661-1672 (2007).

19. Baraffe, I., Chabrier, G. \& Barman, T. Structure and evolution of super-Earth to super-Jupiter exoplanets. I. Heavy element enrichment in the interior. Astron. Astrophys. 482, 315-332 (2008).

20. Emsenhuber, A. et al. The New Generation Planetary Population Synthesis (NGPPS). II. Planetary population of solar-like stars and overview of statistical results. Preprint at https://arxiv.org/abs/2007.05562 (2020).

21. Poppenhaeger, K., Ketzer, L. \& Mallonn, M. X-ray irradiation and evaporation of the four young planets around V1298 Tau. Mon. Not. R. Astron. Soc. 500, 4560-4572 (2021).

22. Thorngren, D. P., Fortney, J. J., Murray-Clay, R. A. \& Lopez, E. D. The mass-metallicity relation for giant planets. Astrophys. J. 831, 64 (2016)

23. Wahl, S. M. et al. Comparing Jupiter interior structure models to Juno gravity measurements and the role of a dilute core. Geophys. Res. Lett. 44, 4649-4659 (2017).

24. Turrini, D. et al. Tracing the formation history of giant planets in protoplanetary disks with carbon, oxygen, nitrogen, and sulfur. Astrophys. J. 909, 40 (2021)

25. Cosentino, R. et al. Harps-N: the new planet hunter at TNG. Proc. SPIE 8446, 84461V (2012).

26. Covino, E. et al. The GAPS programme with HARPS-N at TNG. I. Observations of the Rossiter-McLaughlin effect and characterisation of the transiting system Qatar-1. Astron. Astrophys. 554, A28 (2013).

27. Carleo, I. et al. The GAPS Programme at TNG. XXI. A GIARPS case study of known young planetary candidates: confirmation of HD $285507 \mathrm{~b}$ and refutation of AD Leonis b. Astron. Astrophys. 638, A5 (2020).

28. Noyes, R. W., Hartmann, L. W., Baliunas, S. L., Duncan, D. K. \& Vaughan, A. $\mathrm{H}$. Rotation, convection, and magnetic activity in lower main-sequence stars. Astrophys. J. 279, 763-777 (1984).

29. Gomes da Silva, J. et al. Long-term magnetic activity of a sample of M-dwarf stars from the HARPS program. I. Comparison of activity indices. Astron. Astrophys. 534, A30 (2011).

30. Díaz, R. F., Cincunegui, C. \& Mauas, P. J. D. The Na I D resonance lines in main-sequence late-type stars. Mon. Not. R. Astron. Soc. 378 , 1007-1018 (2007).

31. Azizi, F. \& Mirtorabi, M. T. A survey of TiO $\lambda 567 \mathrm{~nm}$ absorption in solar-type stars. Mon. Not. R. Astron. Soc. 475, 2253-2268 (2018).

32. Quirrenbach, A. et al. CARMENES instrument overview. Proc. SPIE 9147, 91471F (2014)

33. Zechmeister, M., Anglada-Escudé, G. \& Reiners, A. Flat-relative optimal extraction. A quick and efficient algorithm for stabilised spectrographs. Astron. Astrophys. 561, A59 (2014).

34. Raskin, G. et al. HERMES: a high-resolution fibre-fed spectrograph for the Mercator telescope. Astron. Astrophys. 526, A69 (2011).

35. Baranne, A. et al. ELODIE: A spectrograph for accurate radial velocity measurements. Astron. Astrophys. Suppl. Ser. 119, 373-390 (1996).

36. Strassmeier, K. G. et al. The STELLA robotic observatory. Astron. Nachr. 325 527-532 (2004)

37. Collins, K. A., Kielkopf, J. F., Stassun, K. G. \& Hessman, F. V. AstroImageJ: image processing and photometric extraction for ultra-precise astronomical light curves. Astron. J. 153, 77 (2017). 
38. Luger, R., Kruse, E., Foreman-Mackey, D., Agol, E. \& Saunders, N. An update to the EVEREST K2 pipeline: short cadence, saturated stars, and Kepler-like photometry down to Kp=15. Astron. J. 156, 99 (2018).

39. Deming, D. et al. Infrared transmission spectroscopy of the exoplanets HD 209458b and XO-1b using the Wide Field Camera-3 on the Hubble Space Telescope. Astrophys. J. 774, 95 (2013).

40. Luhman, K. L. The stellar membership of the Taurus star-forming region. Astron. J. 156, 271 (2018).

41. Andrews, J. J., Chanamé, J. \& Agüeros, M. A. Wide binaries in Tycho-Gaia: search method and the distribution of orbital separations. Mon. Not. R. Astron. Soc. 472, 675-699 (2017).

42. González Hernández, J. I. et al. The solar gravitational redshift from HARPS-LFC Moon spectra. A test of the general theory of relativity. Astron. Astrophys. 643, A146 (2020).

43. Suárez Mascareño, A. et al. The RoPES project with HARPS and HARPS-N. I. A system of super-Earths orbiting the moderately active K-dwarf HD 176986. Astron. Astrophys. 612, A41 (2018).

44. Foreman-Mackey, D., Hogg, D. W., Lang, D. \& Goodman, J. emcee: the MCMC hammer. Publ. Astron. Soc. Pac. 125, 306 (2013).

45. Allende Prieto, C. et al. A spectroscopic study of the ancient Milky Way: F- and G-type stars in the third data release of the Sloan Digital Sky Survey. Astrophys. J. 636, 804-820 (2006).

46. Allende Prieto, C. et al. A collection of model stellar spectra for spectral types B to early-M. Astron. Astrophys. 618, A25 (2018).

47. Asplund, M., Grevesse, N., Sauval, A. J. \& Scott, P. The chemical composition of the Sun. Annu. Rev. Astron. Astrophys. 47, 481-522 (2009).

48. Kurucz, R. L., Furenlid, I., Brault, J. \& Testerman, L. Solar Flux Atlas from 296 to $1300 \mathrm{~nm}$ (Aura, 1984)

49. Dutra-Ferreira, L., Pasquini, L., Smiljanic, R., Porto de Mello, G. F. \& Steffen, M. Consistent metallicity scale for cool dwarfs and giants. A benchmark test using the Hyades. Astron. Astrophys. 585, A75 (2016).

50. Castelli, F. \& Kurucz, R. L. in Modelling of Stellar Atmospheres Vol. 210 (eds Piskunov, N. et al.) A20 (Astronomical Society of the Pacific, 2003).

51. González Hernández, J. I. \& Bonifacio, P. A new implementation of the infrared flux method using the 2MASS catalogue. Astron. Astrophys. 497, 497-509 (2009).

52. Henden, A. A., Levine, S. E., Terrell, D., Smith, T. C. \& Welch, D. Data Release 3 of the AAVSO All-Sky Photometric Survey (APASS). J. Am. Assoc Var. Star Obs. 40, 430 (2012)

53. Schlegel, D. J., Finkbeiner, D. P. \& Davis, M. Maps of dust infrared emission for use in estimation of reddening and cosmic microwave background radiation foregrounds. Astrophys. J. 500, 525-553 (1998).

54. Bonifacio, P., Monai, S. \& Beers, T. C. A search for stars of very low metal abundance. V. Photoelectric UBV photometry of metal-weak candidates from the northern HK survey. Astron. J. 120, 2065-2081 (2000).

55. Sneden, C. A. Carbon and Nitrogen Abundances in Metal-Poor Stars. PhD thesis, Univ. Texas at Austin (1973).

56. Lind, K., Asplund, M. \& Barklem, P. S. Departures from LTE for neutral Li in late-type stars. Astron. Astrophys. 503, 541-544 (2009).

57. Peacock, M. B., Zepf, S. E. \& Finzell, T. Signatures of multiple stellar populations in unresolved extragalactic globular/young massive star clusters. Astrophys. J. 769, 126 (2013).

58. Bayo, A. et al. VOSA: virtual observatory SED analyzer. An application to the Collinder 69 open cluster. Astron. Astrophys. 492, 277-287 (2008).

59. Baraffe, I., Homeier, D., Allard, F. \& Chabrier, G. New evolutionary models for pre-main sequence and main sequence low-mass stars down to the hydrogen-burning limit. Astron. Astrophys. 577, A42 (2015).

60. Tognelli, E., Prada Moroni, P. G. \& Degl'Innocenti, S. The Pisa pre-main sequence tracks and isochrones. A database covering a wide range of $Z, Y$, mass, and age values. Astron. Astrophys. 533, A109 (2011).

61. Bressan, A. et al. PARSEC: stellar tracks and isochrones with the PAdova and TRieste Stellar Evolution Code. Mon. Not. R. Astron. Soc. 427, 127-145 (2012).

62. del Burgo, C. \& Allende Prieto, C. Testing models of stellar structure and evolution - I. Comparison with detached eclipsing binaries. Mon. Not. R. Astron. Soc. 479, 1953-1973 (2018).

63. Miret-Roig, N. et al. Dynamical traceback age of the $\beta$ Pictoris moving group. Astron. Astrophys. 642, A179 (2020).

64. Rebull, L. M. et al. Rotation of low-mass stars in Taurus with K2. Astron. J. 159, 273 (2020).

65. Dahm, S. E. Reexamining the lithium depletion boundary in the Pleiades and the inferred age of the cluster. Astrophys. J. 813, 108 (2015).

66. Gossage, S. et al. Age determinations of the Hyades, Praesepe, and Pleiades via MESA models with rotation. Astrophys. J. 863, 67 (2018).

67. Gutiérrez Albarrán, M. L. et al. The Gaia-ESO Survey: calibrating the lithium-age relation with open clusters and associations. I. Cluster age range and initial membership selections. Astron. Astrophys. 643, A71 (2020).

68. Barrado y Navascués, D., Stauffer, J. R. \& Patten, B. M. The lithium-depletion boundary and the age of the young open cluster IC 2391. Astrophys. J. 522, L53-L56 (1999).
69. Wichmann, R. et al. New weak-line T Tauri stars in Taurus-Auriga. Astron. Astrophys. 312, 439-454 (1996).

70. Micela, G. et al. Deep ROSAT HRI observations of the Pleiades. Astron. Astrophys. 341, 751-767 (1999).

71. Fang, X.-S., Zhao, G., Zhao, J.-K. \& Bharat Kumar, Y. Stellar activity with LAMOST - II. Chromospheric activity in open clusters. Mon. Not. R. Astron. Soc. 476, 908-926 (2018).

72. Findeisen, K., Hillenbrand, L. \& Soderblom, D. Stellar activity in the broadband ultraviolet. Astron. J. 142, 23 (2011)

73. Foreman-Mackey, D., Agol, E., Ambikasaran, S. \& Angus, R. Fast and scalable Gaussian process modeling with applications to astronomical time series. Astron. J. 154, 220 (2017).

74. Haywood, R. D. et al. Planets and stellar activity: hide and seek in the CoRoT-7 system. Mon. Not. R. Astron. Soc. 443, 2517-2531 (2014).

75. Suárez Mascareño, A. et al. Revisiting Proxima with ESPRESSO. Astron. Astrophys. 639, A77 (2020)

76. Feng, F., Tuomi, M., Jones, H. R. A., Butler, R. P. \& Vogt, S. A Goldilocks principle for modelling radial velocity noise. Mon. Not. R. Astron. Soc. 461, 2440-2452 (2016).

77. Parviainen, H. PYTRANSIT: fast and easy exoplanet transit modelling in PYTHON. Mon. Not. R. Astron. Soc. 450, 3233-3238 (2015).

78. Mandel, K. \& Agol, E. Analytic light curves for planetary transit searches. Astrophys. J. 580, L171-L175 (2002).

79. Fulton, B. J., Petigura, E. A., Blunt, S. \& Sinukoff, E. RadVel: the radial velocity modeling toolkit. Publ. Astron. Soc. Pac. 130, 044504 (2018).

80. Skilling, J. Nested sampling. AIP Conf. Proc. 735, 395-405 (2004).

81. Speagle, J. S. DYNESTY: a dynamic nested sampling package for estimating Bayesian posteriors and evidences. Mon. Not. R. Astron. Soc. 493 3132-3158 (2020).

82. Ambikasaran, S., Foreman-Mackey, D., Greengard, L., Hogg, D. W. \& O’Neil, M. Fast direct methods for Gaussian processes. IEEE Trans. Pattern Anal. Mach. Intell. 38, 252-265 (2015).

83. Benatti, S. et al. Constraints on the mass and atmospheric composition and evolution of the low-density young planet DS Tuc A b. Astron. Astrophys. 650, A66 (2021).

84. Zechmeister, M. \& Kürster, M. The generalised Lomb-Scargle periodogram. A new formalism for the floating-mean and Keplerian periodograms. Astron. Astrophys. 496, 577-584 (2009).

85. Gaia Collaboration et al. Gaia Early Data Release 3. Summary of the contents and survey properties. Astron. Astrophys. 649, A1 (2021).

86. Gaia Collaboration et al. Gaia Data Release 2. Summary of the contents and survey properties. Astron. Astrophys. 616, Al (2018).

87. Nguyen, D. C., Brandeker, A., van Kerkwijk, M. H. \& Jayawardhana, R. Close companions to young stars. I. A large spectroscopic survey in Chamaeleon I and Taurus-Auriga. Astrophys. J. 745, 119 (2012).

88. Nesterov, V. V. et al. The Henry Draper extension charts: a catalogue of accurate positions, proper motions, magnitudes and spectral types of 86933 stars. Astron. Astrophys. Suppl. Ser. 110, 367 (1995).

89. Høg, E. et al. The Tycho- 2 catalogue of the 2.5 million brightest stars. Astron. Astrophys. 355, L27-L30 (2000).

90. Skrutskie, M. F. et al. The Two Micron All Sky Survey (2MASS). Astron. J. 131, 1163-1183 (2006).

\section{Acknowledgements}

A.S.M. acknowledges financial support from the Spanish Ministry of Science and Innovation (MICINN) under the 2019 Juan de la Cierva Programme. J.I.G.H. acknowledges financial support from the Spanish MICINN under the 2013 Ramón y Cajal programme RYC-2013-14875. A.S.M., J.I.G.H., R.R., B.T.-P., N.L., M.R.Z.O., E.G.-A., J.A.C., P.J.A. and I.R. acknowledge financial support from the Spanish Ministry of Science and Innovation through projects AYA2017-86389-P, PID2019-109522GB-C53, PID2019-109522GB-C51, AYA2016-79425-C3-3-P, PID2019-109522GB-C52 and PGC2018-098153-B-C33. M.D. acknowledges financial support from the FP7-SPACE Project ETAEARTH (GA no. 313014). A.M., D.L., G.M., A.S. and S.D. acknowledge partial contribution from the agreement ASI-INAF no. 2018-16-HH.0. S.B., D.L., G.M. and D.T. acknowledge partial contribution from the agreement ASI-INAF no. 20215-HH.0. P.J.A. acknowledges financial support from the project SEV-2017-0709. S.D., V.D., S.B. and D.T. acknowledge support from the PRIN-INAF 2019 'Planetary systems at young ages' (PLATEA). D.S.A. thanks the Leverhulme Trust for financial support. I.R. acknowledges the support of the Generalitat de Catalunya/CERCA programme. E.G.-A acknowledges support from the Spanish State Research Agency (AEI) project no. MDM-2017-0737 Unidad de Excelencia 'María de Maeztu' Centro de Astrobiología (CAB, CSIC/INTA). D.T. acknowledges the support of the Italian National Institute of Astrophysics (INAF) through the INAF Main Stream project 'Ariel and the astrochemical link between circumstellar discs and planets' (CUP: C54I19000700005). E.E.-B. acknowledges financial support from the European Union and the State Agency of Investigation of the Spanish Ministry of Science and Innovation (MICINN) under grant PRE2020-093107 of the Pre-Doc Program for the Training of Doctors (FPI-SO) through FEDER, FSE and FDCAN funds. This work is based on observations made with the Italian Telescopio Nazionale Galileo (TNG) operated by the Fundación Galileo Galilei 
(FGG) of the Istituto Nazionale di Astrofisica (INAF) at the Observatorio del Roque de los Muchachos (La Palma, Canary Islands, Spain). CARMENES is an instrument at the Centro Astronómico Hispano-Alemán (CAHA) at Calar Alto (Almería, Spain), operated jointly by the Junta de Andalucía and the Instituto de Astrofísica de Andalucía (CSIC).

This work is based on data obtained with the STELLA robotic telescopes in Tenerife, an AIP facility jointly operated by AIP and IAC. This work makes use of observations from the LCOGT network. This work is based on observations made with the Nordic Optical Telescope, owned in collaboration by the University of Turku and Aarhus University, and operated jointly by Aarhus University, the University of Turku and the University of Oslo (representing Denmark, Finland and Norway), the University of Iceland and Stockholm University; the telescope is located at the Observatorio del Roque de los Muchachos, La Palma, Spain, of the Instituto de Astrofisica de Canarias.

\section{Author contributions}

A.S.M. wrote the main text of the manuscript. A.S.M., V.J.S.B., J.I.G.H., M.R.Z.O. and C.d.B. wrote the methods section of the manuscript. A.S.M. and M.D. performed the radial velocity analysis. N.L., A.S., V.J.S.B., G.M., R.R., S.B., C.C.G., G.N., R.L., E.P., A.S.M., M.D., P.J.A., E.G.-A. and M.W. coordinated the acquisition of the radial velocities. V.J.S.B., F. Murgas, E.P., H.P., E.E.-B. and M.M. coordinated the acquisition of the photometry. A.S.M., B.T.-P., F.F.B. and T.G. performed the extraction of radial velocities. F. Murgas performed the extraction of the photometry. V.J.S.B., M.R.Z.O., J.I.G.H., C.d.B., H.M.T., D.S.A., N.L., E.L.M. and P.C. determined the stellar properties of V1298 Tau and HD 284154. R.C., A.M. and D.T. contributed to the discussion on planetary evolution. R.R., A.S., M.R.Z.O., V.J.S.B. and G.M. organized the collaboration between the different teams. M.D., A.S., S.B., G.M., S.D., R.C., L.M., V.D., D.L., F. Marzari, D.T. and A.M. are members of the GAPS consortium. P.J.A., J.A., J.A.C., A.Q., A.R., I.R., M.R.Z.O., V.J.S.B., J.I.G.H., N.L, G.N., R.L., E.P., M.O., E.L.M. and R.R. are members of the CARMENES consortium. L.M. participated in the discussion of stellar activity. T.G., K.G.S. and M.W. are members of the STELLA consortium. All authors were given the opportunity to review the results and comment on the manuscript.

\section{Competing interests}

The authors declare no competing interests.

\section{Additional information}

Extended data is available for this paper at https://doi.org/10.1038/s41550-021-01533-7. Supplementary information The online version contains supplementary material available at https://doi.org/10.1038/s41550-021-01533-7.

Correspondence and requests for materials should be addressed to A. Suárez Mascareño. Peer review information Nature Astronomy thanks the anonymous reviewers for their contribution to the peer review of this work

Reprints and permissions information is available at www.nature.com/reprints.

Publisher's note Springer Nature remains neutral with regard to jurisdictional claims in published maps and institutional affiliations.

(C) The Author(s), under exclusive licence to Springer Nature Limited 2021 


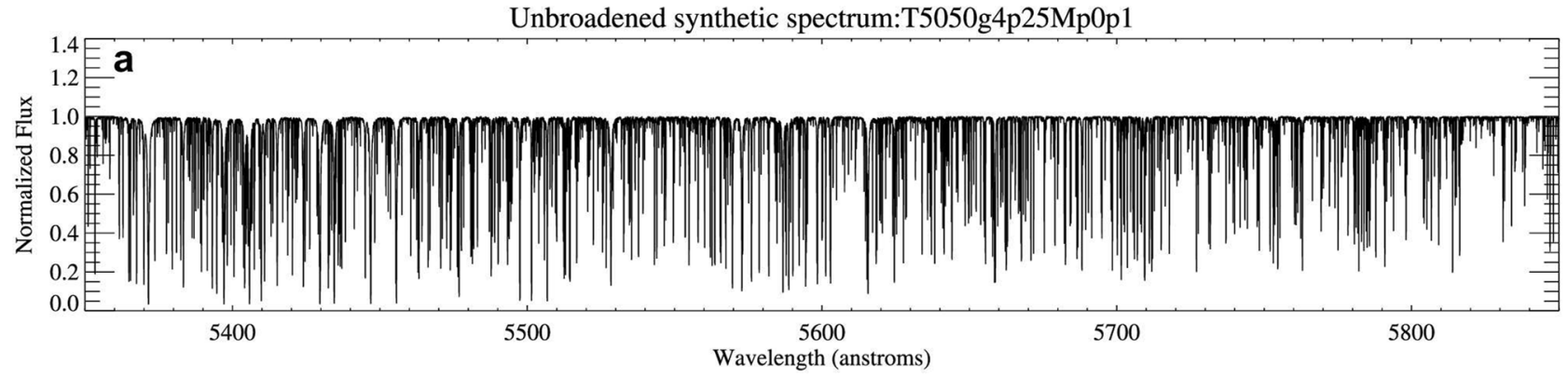

Broadened synthetic spectrum

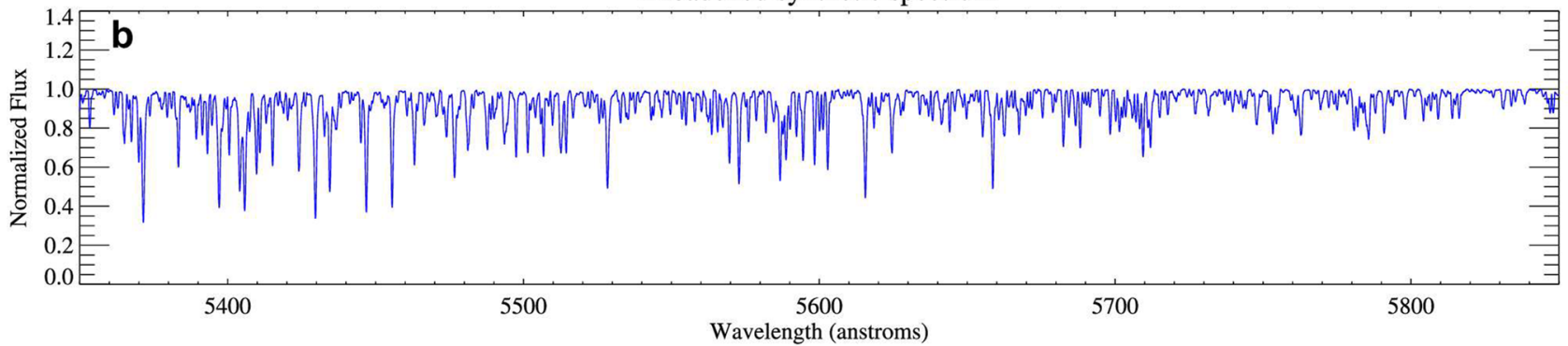

V1298 Tau: observed and synthetic spectra

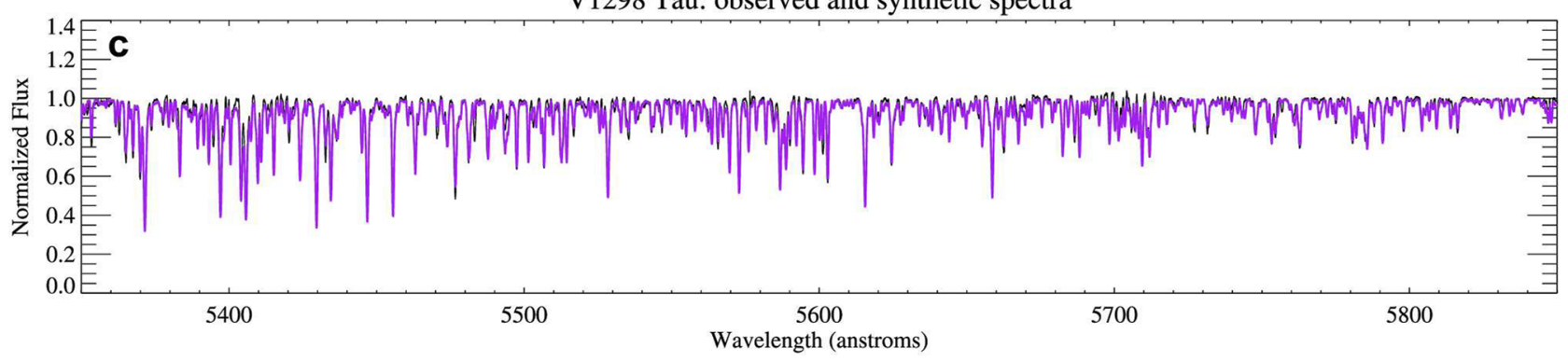

Extended Data Fig. 1 | Best synthetic spectral fit of the HARPS-N spectrum of V1298 Tau. The interpolated SYNPLE synthetic spectrum without rotational broadening computed for the derived best-fit stellar parameters and metallicity (a), the broadened spectrum with a rotational velocity of 24 $\mathrm{km} \mathrm{s}^{-1}(\mathrm{~b})$ and the observed HARPS-N 1D spectrum of V1298 Tau (black line) together with the best-fit synthetic spectrum (purple line) are displayed in the spectral range 5350-5850 ̊ (c). 


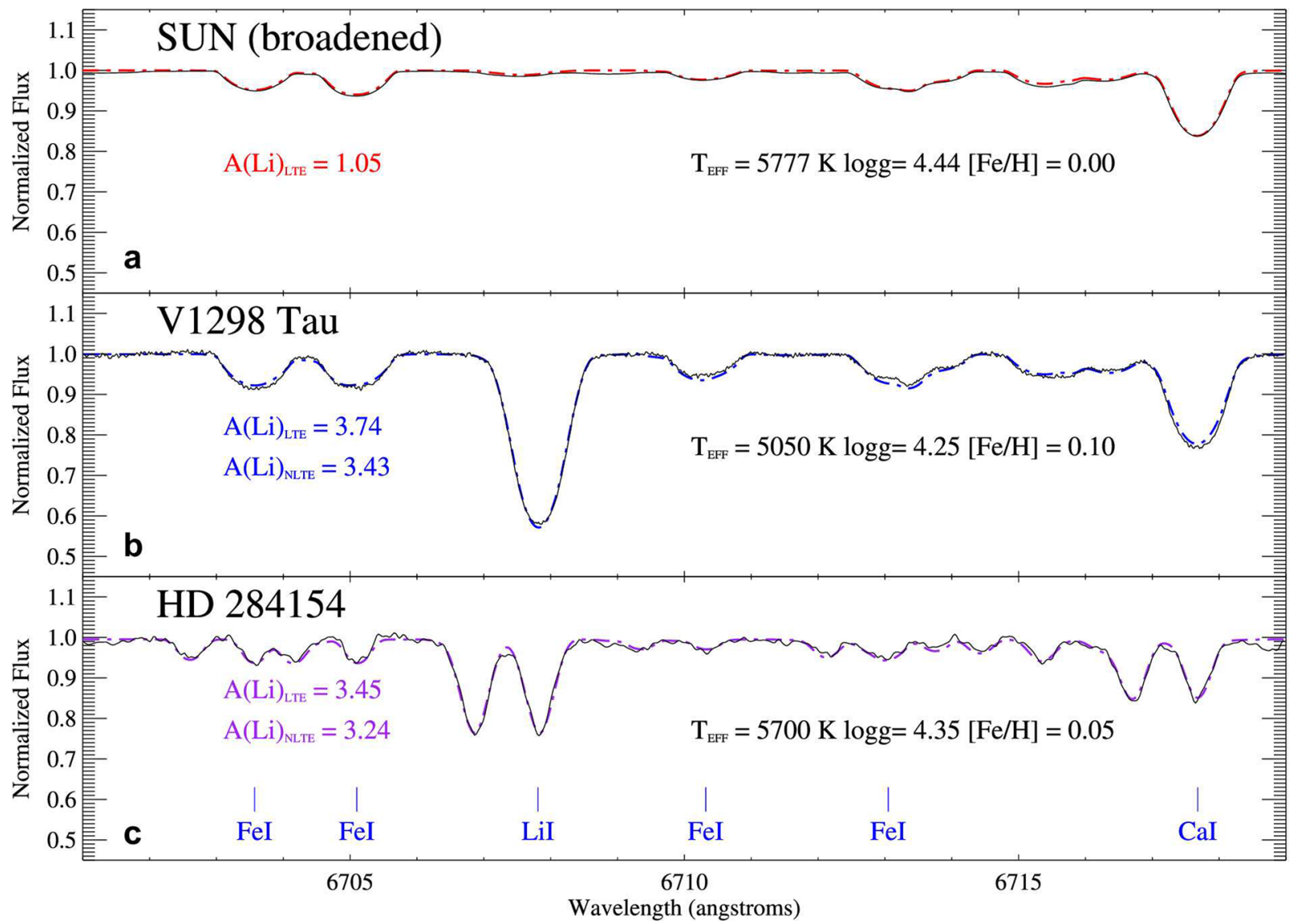

Extended Data Fig. 2 | The lithium spectral region of V1298 Tau and HD 284154. Spectral region of the lithium doublet around $6708 \AA \AA$ of the solar ATLAS spectrum broadened with a rotation profile of $24 \mathrm{~km} \mathrm{~s}^{-1}$ (a), the HARPS-N spectrum of V1298 Tau (b), and the FIES spectrum of the double-lined spectroscopic binary HD 284154 (c), together with the best-fit MOOG synthetic spectra. 

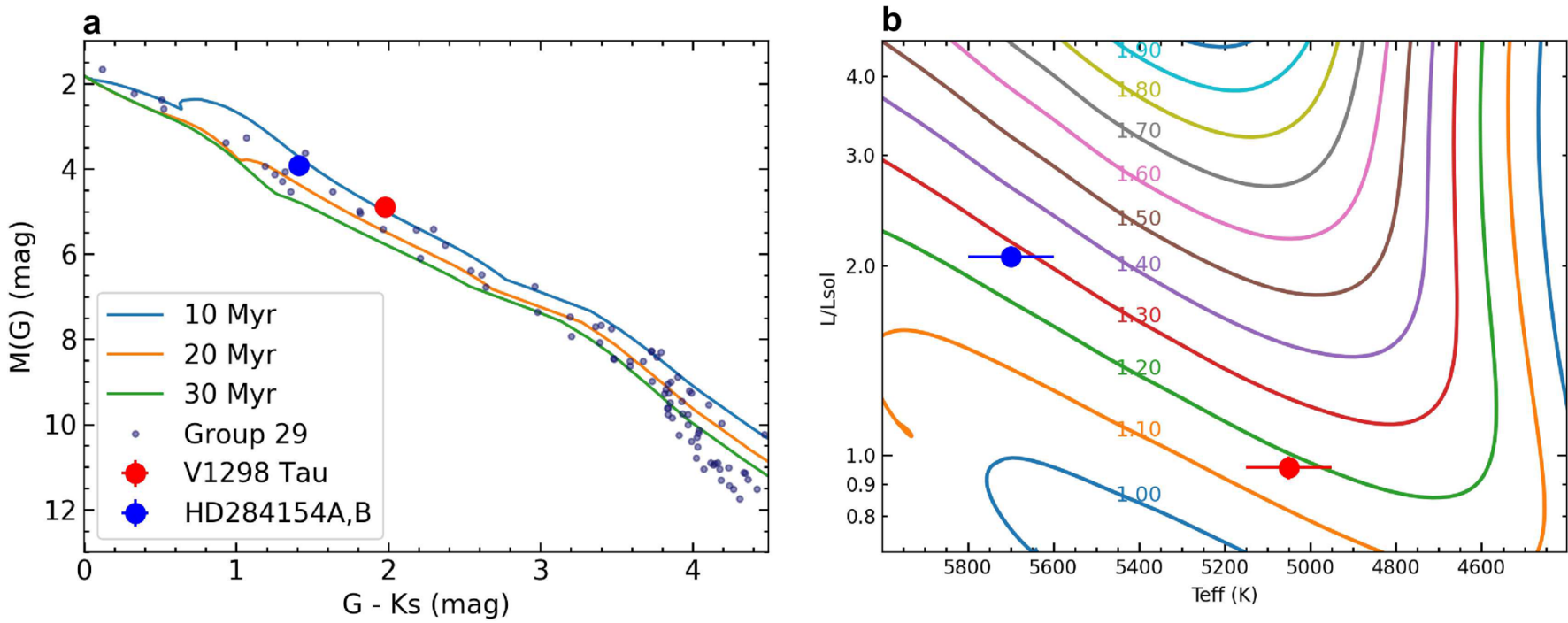

Extended Data Fig. 3 | Position of V1298 Tau and HD 284154 in the colour-magnitude and Hertzsprung-Russel diagrams. a: Colour-magnitude diagram of V1298 Tau and HD 284154A and B (separate components) and the other group 29 members along with various PARSEC isochrones. The 20-Myr isochrone nicely reproduces the sequence of stars with colours $G-K_{s}<3.5$ mag while the 10- and 30-Myr isochrones provide acceptable upper and lower envelopes to the observed dispersion of the Group 29 sequence. b: Location of V1298 Tau (red) and HD 284154 (blue) in the Hertzsprung-Russel diagram. HD 284154 is decomposed into two equal mass and equal luminosity stars. The tracks for masses between 1.0 and $1.9 \mathrm{M}_{\odot}$ are also shown and are labeled with the mass value in solar units. Note that the luminosity axis is in logarithmic scale. The error bar in luminosity is of the size of the symbol. 

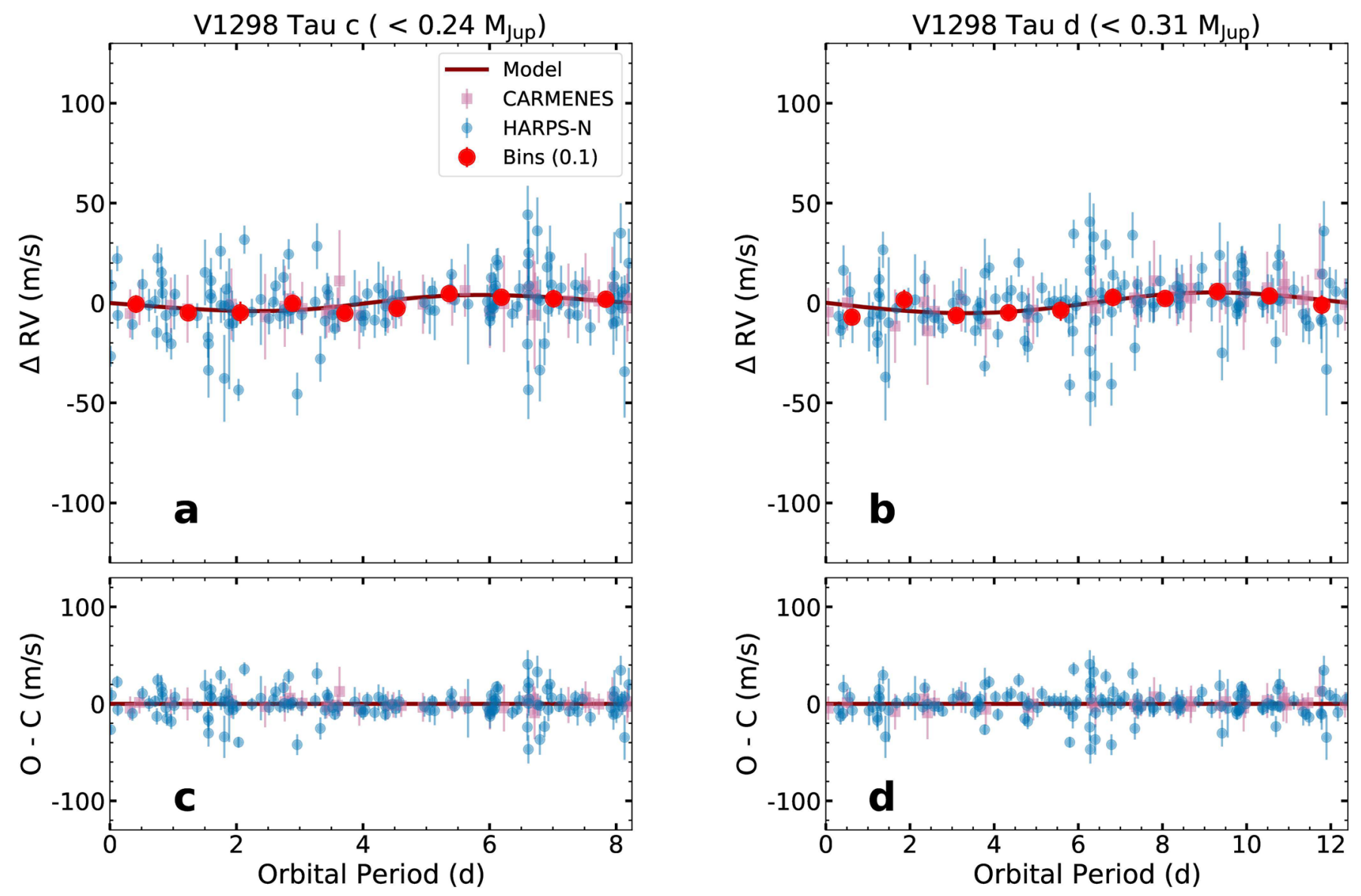

Extended Data Fig. 4 | Phase-folded plots of the RV signals for the two planets of the V1298 Tau planetary system for which we could not confirm the RV signals. a: Phase-folded representation of the best-fitting Keplerian orbit (red line) for V1298 Tau c. b: Same for V1298 Tau d. c and d: Residuals after the fit for both cases. For a better visualisation, only HARPS and CARMENES data have been included. In all cases, $1 \sigma$ error bars (internal RV uncertainties) of the measurements are shown. 

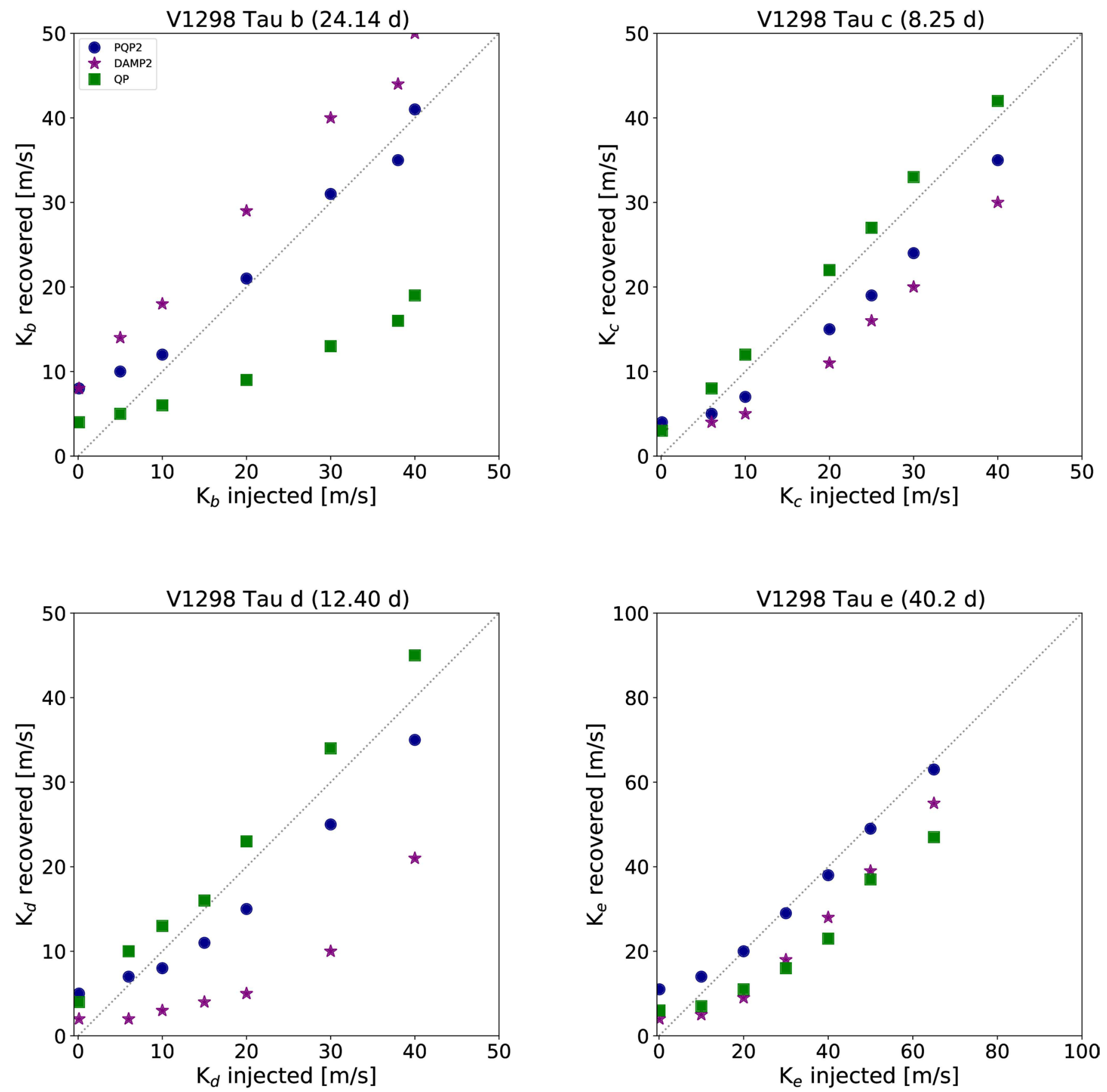

Extended Data Fig. 5 | Accuracy of the recovered planetary amplitudes of the different methods. Recovered planetary amplitude against injected planetary amplitude in the simulated datasets for the four planets in the system. 


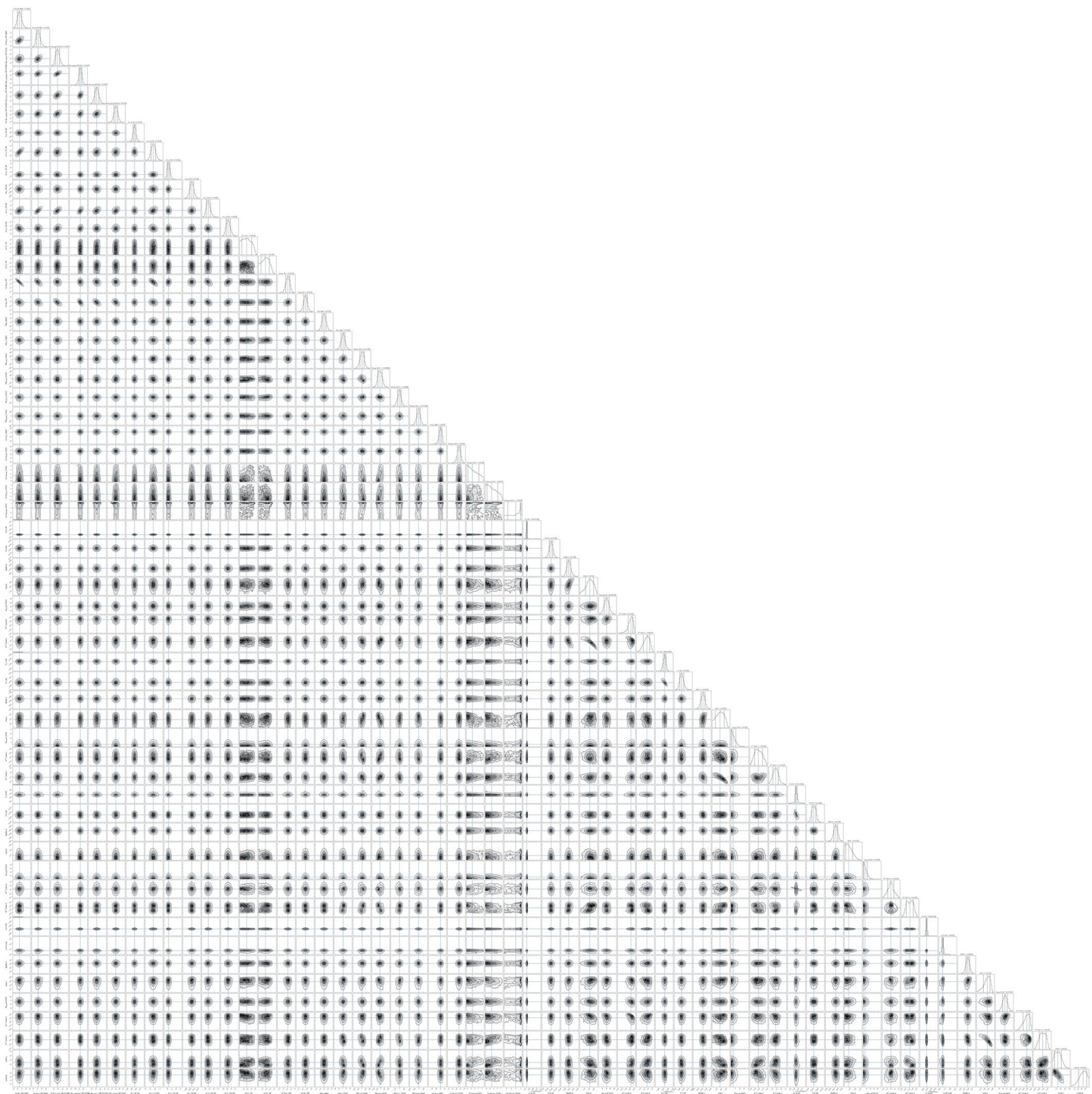

Extended Data Fig. 6 | Corner plot of the parameters of the best model fit (PQP2). Posterior distributions of all the parameters sampled for the best model fit along with the correlation maps between them. 

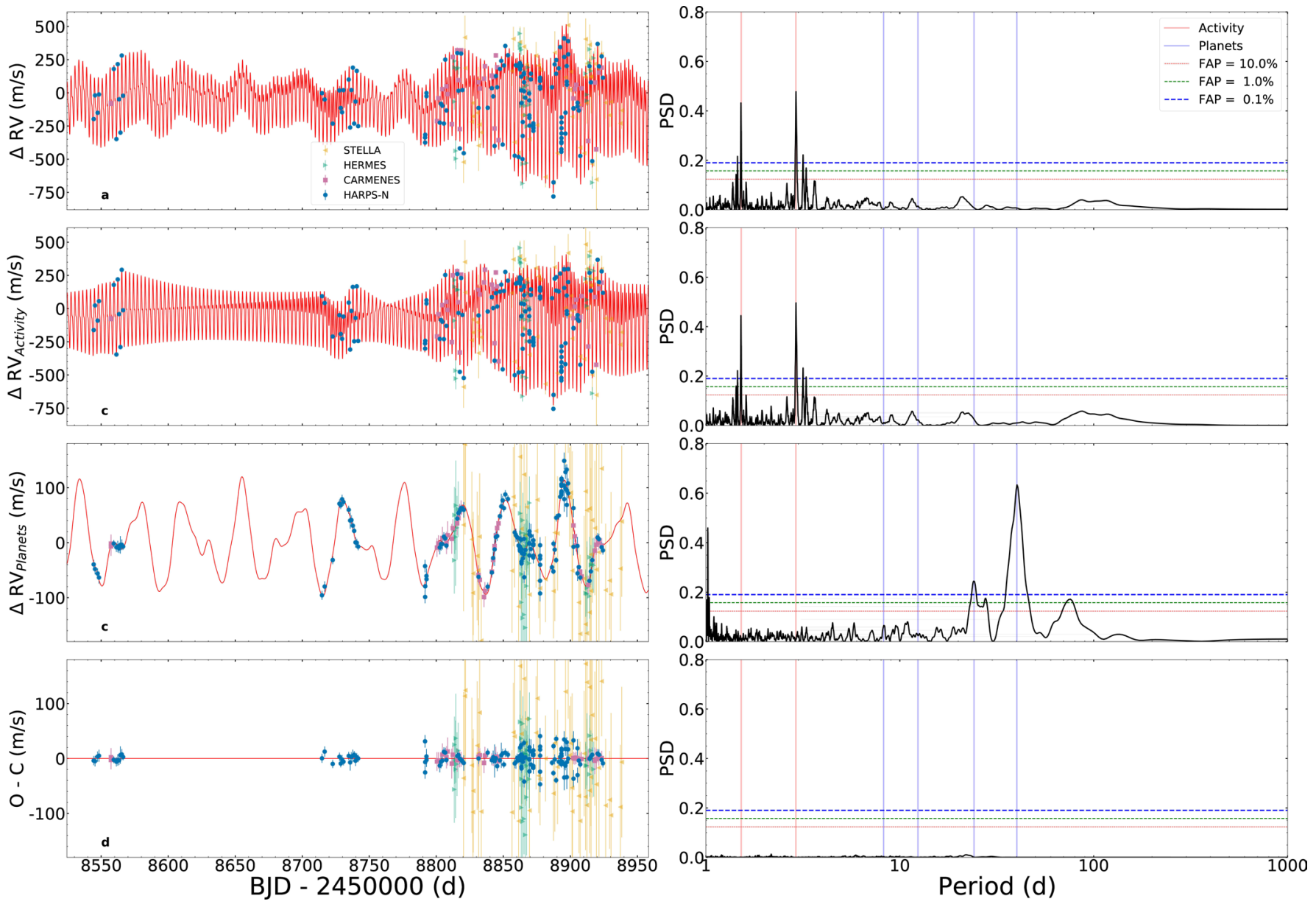

Extended Data Fig. 7 | RV time series with the best model fit of V1298 Tau and their periodograms. $a$ : Full time series with the best fit model combining stellar activity and planetary signals. The stellar activity model represented is a weighted average of the models used for the different spectral ranges. $b$ : Activity induced RV after subtracting the planetary signal. c: Planetary RV component, after subtracting the stellar induced signal. $d$ : Residuals after the fit. $1 \sigma$ error bars (internal RV uncertainties) of the measurements are shown. The right panel of each figure shows the periodogram of the data with their associated levels of false alarm probability. The positions of the activity, and planetary, signals are indicated with red and blue vertical lines, respectively. 


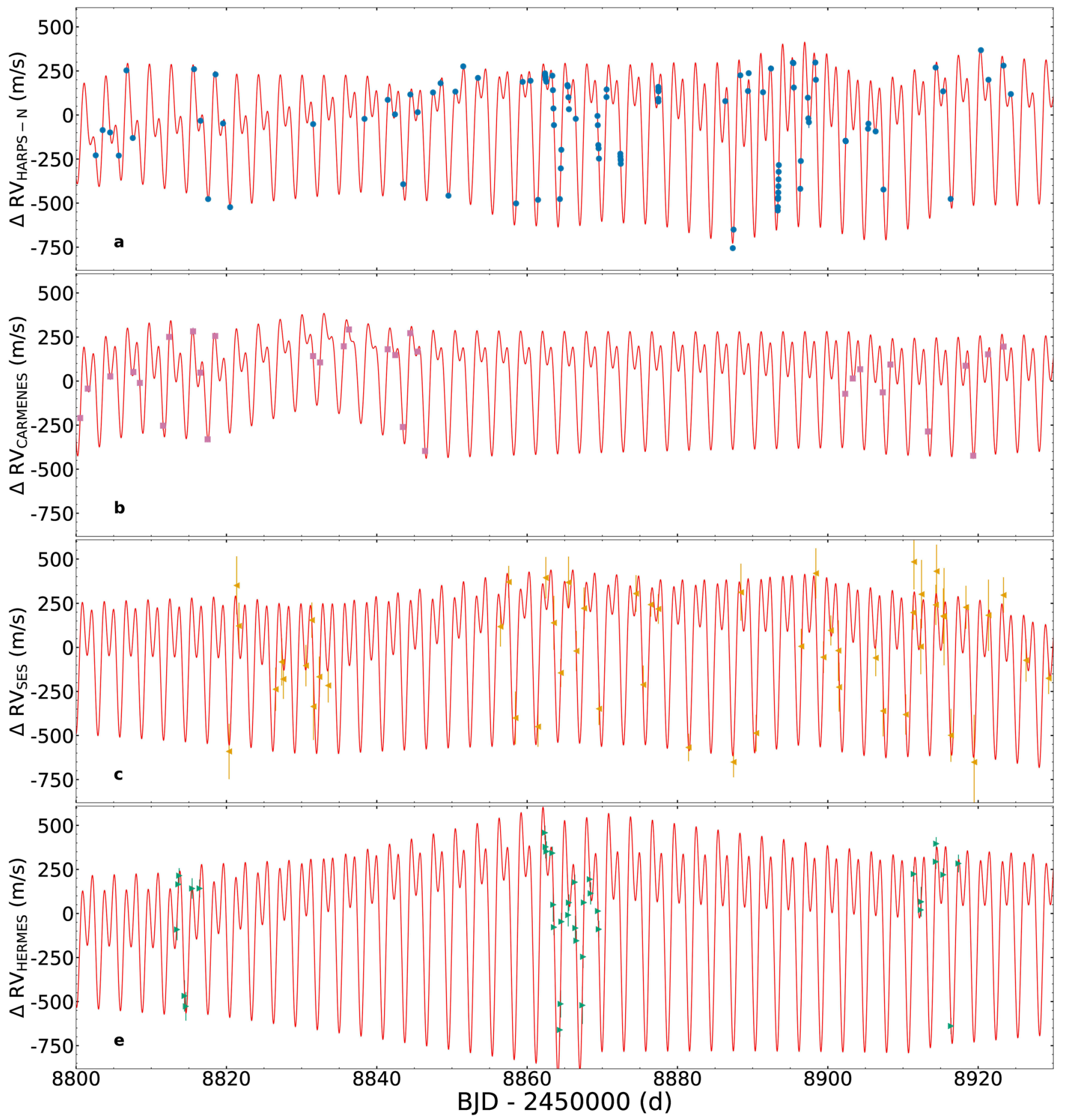

Extended Data Fig. 8 | RV time series of the activity component of V1298 Tau for all instruments. Zoom to the section of the campaign with the largest density of observations. Panel a shows the HARPS-N data after subtracting the planetary component along with the best fit model for the activity component. Panels $b, c$ and $d$ show the CARMENES data, SES data and HERMES data, respectively. $1 \sigma$ error bars (internal RV uncertainties) of the measurements are shown. 

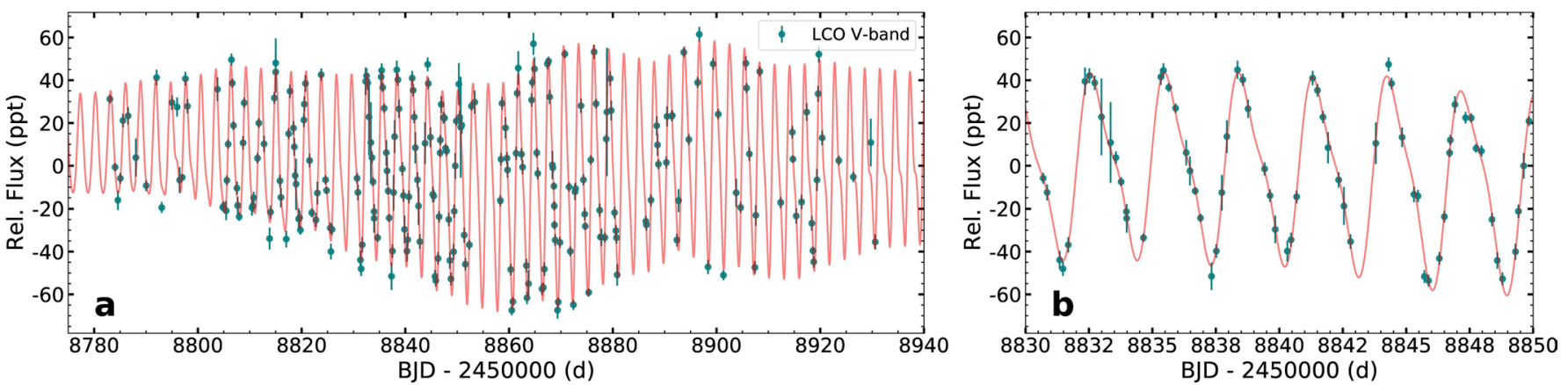

Extended Data Fig. 9 | LCOGT $\boldsymbol{V}$-band photometry. Time series of the LCOGT $\boldsymbol{V}$-band photometry with the best fit obtained from the global analysis. $b$ : Zoom to a well-sampled section. $1 \sigma$ error bars (internal uncertainties) of the measurements are shown. 

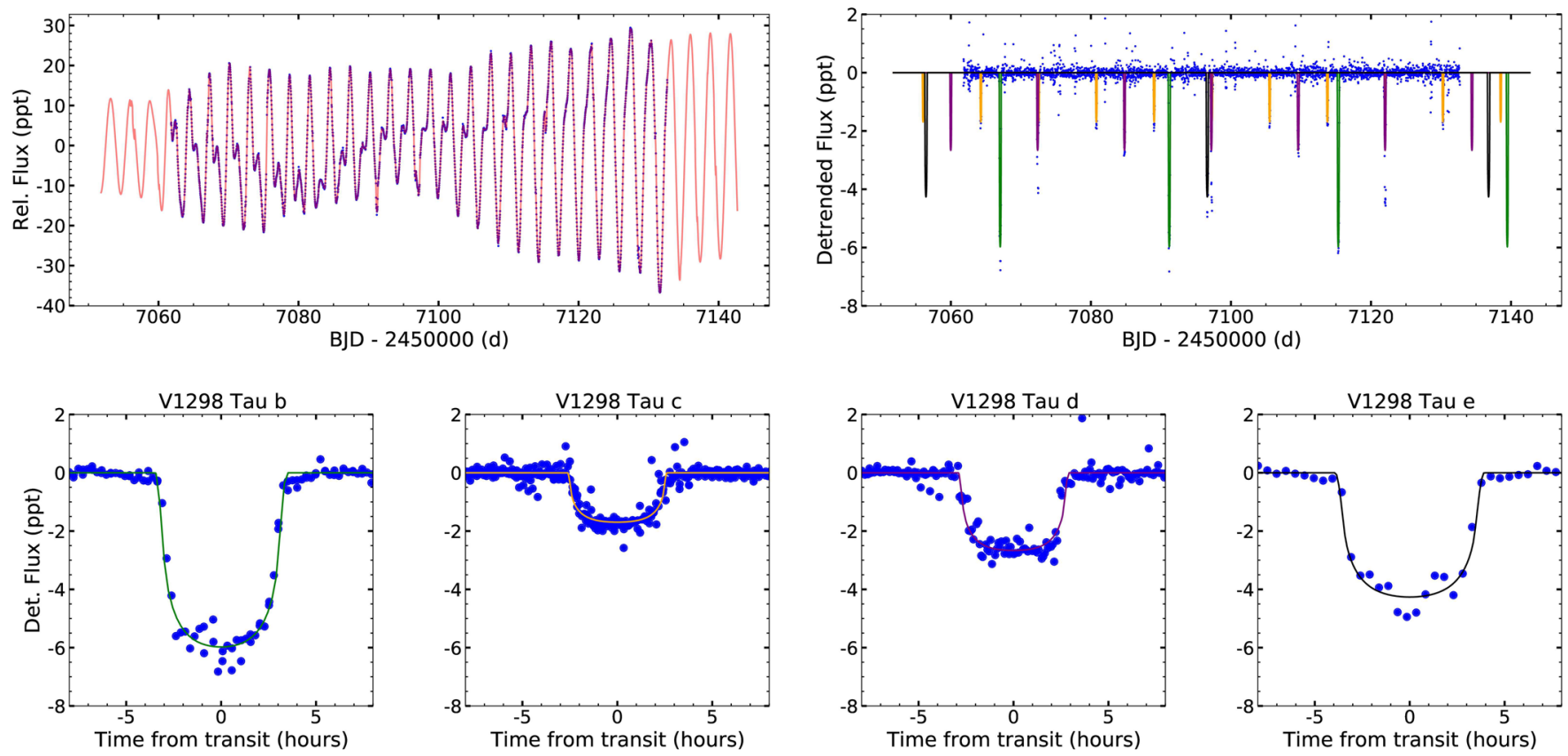

Extended Data Fig. 10 | K2 photometry. Time series of the K2 photometry with the best fit obtained from the global analysis. $a$ : K2 data with the full fit. $b$ : Data detrended from stellar activity with the best fit to the transits. $c, d, e$ and $f$ : Phase-folded plots of the transits of the four planets. 